\title{
Standard model matrix elements for neutral $B$-meson mixing and associated decay constants
}

\author{
Laurent Lellouch \\ LAPTH, Chemin de Bellevue, B.P. 110, F-74941 Annecy-le-Vieux Cedex, France \\ and \\ Centre de Physique Théorique, Case 90\%, CNRS Luminy, F-13288 Marseille Cedex 9, France \\ C.-J. David Lin \\ Department of Physics and Astronomy, The University of Southampton, Southampton SO17 1BJ, England
}

(UKQCD Collaboration)

\begin{abstract}
We present results of quenched lattice calculations of the matrix elements relevant for $B_{d}-\bar{B}_{d}$ and $B_{s}-\bar{B}_{s}$ mixing in the standard model. Results for the corresponding $S U(3)$-breaking ratios, which can be used to constrain or determine $\left|V_{t d}\right|$, are also given. The calculations are performed at two values of the lattice spacing, corresponding to $\beta=6.0$ and $\beta=6.2$, with quarks described by a mean-field-improved Sheikholeslami-Wohlert action. As a by-product, we obtain the leptonic decay constants of $B$ and $D$ mesons. We also present matrix elements relevant for $D^{0}-\bar{D}^{0}$ mixing. Our results are summarized in the Introduction.
\end{abstract}

12.38.Gc, $12.15 . \mathrm{Hh}, 14.40 . \mathrm{Nd}$

\section{INTRODUCTION}

The study of $B_{d}-\bar{B}_{d}$ oscillations enables measurement of the magnitude of the poorly known Cabibbo-KobayashiMaskawa $(\mathrm{CKM})$ matrix element, $V_{t d}$, and thus the determination of one of the sides of the unitarity triangle. The frequency of these oscillations is given by the mass difference,

$$
\Delta m_{d} \equiv M_{B_{d}}^{H}-M_{B_{d}}^{L},
$$

where $M_{B_{d}}^{H}$ and $M_{B_{d}}^{L}$ are the heavy and light mass eigenvalues of the $B_{d}-\bar{B}_{d}$ system. $\Delta m_{d}$ is experimentally measurable from tagged $B_{d}$ meson samples. If It is also calculable in the standard model. Keeping only dimension six operators after an operator product expansion in which the top quark and $W$ boson are integrated out, the standard model prediction for $\Delta m_{d}$ is, to next to leading order (NLO) [3, 何:

$$
\Delta m_{d}=\frac{G_{F}^{2}}{8 \pi^{2}} M_{W}^{2}\left|V_{t d} V_{t b}^{*}\right|^{2} \eta_{B} S_{0}\left(x_{t}\right) C_{B}(\mu) \frac{\left|\left\langle\bar{B}_{d}\left|\mathcal{O}_{d}^{\Delta B=2}(\mu)\right| B_{d}\right\rangle\right|}{2 M_{B_{d}}},
$$

where $x_{t}=m_{t}^{2} / M_{W}^{2}, S_{0}\left(x_{t}\right) \simeq 0.784 x_{t}^{0.76}$ (to better than 1\%) is the relevant Inami-Lim function [5], $\mu$ the renormalization scale, $\mathcal{O}_{d}^{\Delta B}=2$ the four-quark operator $\left[\bar{b} \gamma^{\mu}\left(1-\gamma^{5}\right) d\right]\left[\bar{b} \gamma_{\mu}\left(1-\gamma^{5}\right) d\right]$ and $\eta_{B}=0.55$ and $C_{B}(\mu)$, short-distance coefficients. The renormalization-scale dependence of $C_{B}(\mu)$ and of the hadronic matrix element cancel such that $\Delta m_{d}$ is $\mu$-independent, to the order in perturbation theory at which $C_{B}(\mu)$ is calculated. In the naive dimensional regularization modified minimal subtraction (NDR- $\overline{\mathrm{MS}}$ ) scheme assumed here,

$$
C_{B}(\mu)=\left[\alpha_{s}(\mu)\right]^{-6 / 23}\left[1+\frac{\alpha_{s}(\mu)}{4 \pi} J_{5}\right], \quad J_{5}=\frac{5165}{3174} .
$$

Since $\left|V_{t b}\right|$ is equal to unity to very good accuracy, a measurement of $\Delta m_{d}$ clearly enables the determination of $\left|V_{t d}\right|$. The accuracy of this determination is limited, at present, by the theoretical uncertainty in the calculation of the nonperturbative, strong-interaction effects in the matrix element $\left\langle\bar{B}_{d}\left|\mathcal{O}_{d}^{\Delta} B=2(\mu)\right| B_{d}\right\rangle$.

An alternative approach, in which many theoretical uncertainties cancel, is to consider the ratio, $\Delta m_{s} / \Delta m_{d}$, where $\Delta m_{s}$ is the mass difference in the neutral $B_{s}-\bar{B}_{s}$ system. In the standard model,

\footnotetext{
${ }^{1}$ For a recent experimental review see, for instance, [1] or [2].
} 


$$
\frac{\Delta m_{s}}{\Delta m_{d}}=\left|\frac{V_{t s}}{V_{t d}}\right|^{2} \frac{M_{B_{s}}}{M_{B_{d}}} \xi^{2}=\left|\frac{V_{t s}}{V_{t d}}\right|^{2} \frac{M_{B_{d}}}{M_{B_{s}}} r_{s d} \equiv\left|\frac{V_{t s}}{V_{t d}}\right|^{2} \frac{M_{B_{d}}}{M_{B_{s}}}\left|\frac{\left\langle\bar{B}_{s}\left|\mathcal{O}_{s}^{\Delta B=2}\right| B_{s}\right\rangle}{\left\langle\bar{B}_{d}\left|\mathcal{O}_{d}^{\Delta B=2}\right| B_{d}\right\rangle}\right|
$$

where $\mathcal{O}_{s}^{\Delta B=2}$ is the same operator as $\mathcal{O}_{d}^{\Delta B=2}$, with $d$ replaced by $s$, and where we have omitted the renormalizationscale dependence, as it cancels in the ratio. Because the unitarity of the CKM matrix implies $\left|V_{t s}\right| \simeq\left|V_{c b}\right|$ to a few percent and a clean extraction of $\left|V_{c b}\right|$ can be achieved by analyzing semileptonic $B$ decays $[6]$, a measurement of $\Delta m_{s} / \Delta m_{d}$ yields a determination of $\left|V_{t d}\right|$. The high frequency of $B_{s}-\bar{B}_{s}$ oscillations makes this a challenging measurement. Nevertheless, the experimental lower bounds obtained on $\Delta m_{s}$ [1],2] already yield interesting constraints on the unitarity triangle [1, 1 , 12].

The matrix elements that appear in Eq. (1) are traditionally parametrized as

$$
\left\langle\bar{B}_{q}\left|\mathcal{O}_{q}^{\Delta B=2}(\mu)\right| B_{q}\right\rangle=\frac{8}{3} M_{B_{q}}^{2} f_{B_{q}}^{2} B_{B_{q}}(\mu),
$$

where $q=d$ or $s$ and where the parameter, $B_{B_{q}}(\mu)$, measures deviations from vacuum saturation, corresponding to $B_{B_{q}}(\mu)=1$. Here, $f_{B_{q}}$ is the decay constant defined by

$$
\left\langle 0\left|\bar{b} \gamma_{\mu} \gamma^{5} q\right| B_{q}(\vec{p})\right\rangle=i p_{\mu} f_{B_{q}} .
$$

One also usually introduces a renormalization-group invariant and scheme-independent parameter, $\hat{B}_{B_{q}}$, which to NLO in QCD is given by

$$
\hat{B}_{B_{q}}^{\text {nlo }}=C_{B}(\mu) B_{B_{q}}(\mu),
$$

where $C_{B}(\mu)$ is given by Eq. (3) as long as $B_{B_{q}}(\mu)$ is computed in the NDR-MS scheme with five active quarks. For consistency with the value of $\eta_{B}$ given after Eq. (2),$\alpha_{s}$ should be taken to have its two-loop value with $\Lambda_{\overline{\mathrm{MS}}}^{(5)}=225 \mathrm{MeV}$.

In the present paper we report on high statistics, quenched lattice QCD calculations of matrix elements and the corresponding $S U(3)$-breaking ratios relevant for neutral $B$-meson mixing. We obtain the $S U(3)$-breaking ratio $r_{s d}$ in two ways: (1) by calculating $B_{B_{s}} / B_{B_{d}}$ and $f_{B_{s}} / f_{B_{d}}$ and combining these two ratios with the experimental mass ratio, $M_{B_{s}} / M_{B_{d}}$ ("indirect" method); (2) by calculating the matrix elements, $\left\langle\bar{B}_{d, s}\left|\mathcal{O}_{d, s}^{\Delta B=2}\right| B_{d, s}\right\rangle$, directly and taking their ratio ("direct" method), as suggested in Ref. 13 . They mainly differ in the required light- and heavy-quark-mass interpolations and extrapolations, since for the "direct" method it is the matrix element and corresponding ratio that are interpolated and extrapolated, while for the "indirect" method it is the $B$-parameters, decay constants, and corresponding ratios.

As described in more detail in Section II, these calculations are performed at two values of the lattice spacing, $a$ $\left(\sim(2.0 \mathrm{GeV})^{-1}\right.$ and $\left.\sim(2.7 \mathrm{GeV})^{-1}\right)$, with relativistic Wilson fermions. In order to keep discretization errors in check, the lattice calculation is performed with heavy quarks whose masses are around that of the charm and the results are extrapolated to the mass of the $b$. Even in the charm sector, however, quarks have compton wavelengths that are not much larger than our lattice spacings, and it is important to reduce discretization errors as much as possible. We attempt to do so by describing quarks with mean-field-improved [14], Sheikholeslami-Wohlert (SW) actions [15]. When combined with improved operators, these actions lead to discretization errors, which are formally smaller than those generated with an unimproved Wilson action $\left(\mathcal{O}\left(\alpha_{s} a\right)\right.$ instead of $\left.\mathcal{O}(a)\right)$, and which may be numerically smaller than those brought about by a tree-level-improved SW action. It is important to note that, as far as four-quark operators are concerned, nonperturbative $\mathcal{O}(a)$-improvement has not yet been undertaken, and all lattice calculations of $B_{d, s}-\bar{B}_{d, s}$ mixing matrix elements have, as we do, $\mathcal{O}\left(\alpha_{s} a\right)$ discretization errors, or worse, $\mathcal{O}(a)$ errors. It is also important to remember that $\mathcal{O}\left(a^{2}\right)$ errors can be significant in the presence of heavy quarks in a relativistic approach.

Alternatively, one could take an effective theory approach and work with static, nonrelativistic QCD (NRQCD) or Fermilab quarks. In these approaches, the matrix elements are expanded in inverse powers of the heavy-quark mass to remove it from the long-distance dynamics. One important advantage is that discretization errors are no longer

\footnotetext{
${ }^{2}$ Our method differs slightly from that of Ref. [13] in that we actually calculate $\left(\left\langle\bar{B}_{s}\left|\mathcal{O}_{s}^{\Delta B}=2\right| B_{s}\right\rangle /\left\langle\bar{B}_{d}\left|\mathcal{O}_{d}^{\Delta B}=2\right| B_{d}\right\rangle\right) \times$ $\left(M_{B_{d}} / M_{B_{s}}\right)$ and multiply by the experimentally measured value of $M_{B_{s}} / M_{B_{s d}}$.
} 
enhanced by this mass. An accurate description of the physics of the $b$ quark, however, requires one to consider corrections in inverse powers of $m_{b}$ and the calculation of these corrections is made difficult by contributions proportional to inverse powers of $a$. The effective theory and relativistic approaches should thus be viewed as complementary.

An additional feature of our calculation is that we extrapolate $S U(3)$-breaking ratios in the heavy-quark mass directly instead of first extrapolating numerator and denominator and then taking their ratio. The heavy-quark-mass dependence will cancel partially between numerator and denominator and therefore make the extrapolation more reliable. This approach turns out to be particularly fruitful for the determination of $f_{B_{s}} / f_{B}$, where the statistical error is significantly reduced by a direct extrapolation of the ratio.

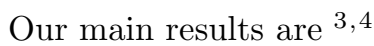

$$
\begin{gathered}
\xi=\frac{f_{B_{s}} \sqrt{B_{B_{s}}}}{f_{B_{d}} \sqrt{B_{B_{d}}}}=1.15(2)_{-2}^{+4}, \quad r_{s d}=\left(\frac{M_{B_{s}}}{M_{B_{d}}}\right)^{2} \xi^{2}=1.38(6)_{-6}^{+10}, \\
f_{B_{d}} \sqrt{\hat{B}_{B_{d}}^{\text {nlo }}}=210(21)_{-26}^{+27} \mathrm{MeV}, \quad \frac{f_{B_{d}}}{f_{D_{s}}} \sqrt{\hat{B}_{B_{d}}^{\text {nlo }}}=0.89(7)_{-7}^{+6}, \\
f_{B_{s}} \sqrt{\hat{B}_{B_{s}}^{\text {nlo }}}=241(14)_{-27}^{+30} \mathrm{MeV}, \quad \frac{f_{B_{s}}}{f_{D_{s}}} \sqrt{\hat{B}_{B_{s}}^{\text {nlo }}}=1.02(4)_{-7}^{+6}, \\
B_{B_{d}}\left(M_{B}\right)=0.91(4)_{-0}^{+4}, \quad \hat{B}_{B_{d}}^{\text {nlo }}=1.40(5)_{-1}^{+6}, \\
B_{B_{s}}\left(M_{B}\right)=0.90(2)_{-0}^{+3}, \quad \hat{B}_{B_{s}}^{\text {nlo }}=1.38(3)_{-0}^{+5}, \\
B_{B_{s}} / B_{B_{d}}=0.98(2)_{-2}^{+0},
\end{gathered}
$$

where the first error is statistical and the second corresponds to the systematic uncertainties added in quadrature. In quantities involving ratios of $B$ parameters, the renormalization-scale dependence is not specified, as it cancels. We consider a wide array of systematic uncertainties, as discussed in Section VII. We normalize dimensionful quantities, involving decay constants, by $f_{D_{s}}$ because some systematic (and statistical) uncertainties, including possibly those associated with quenching, partially cancel in the ratio. The original quantities can then be recovered by using the experimental measurement of $f_{D_{s}}$.

The results of Eq. (8) will be compared, in Section VIII, to earlier calculations of some or all of these quantities performed with propagating heavy quarks [13, 23, 24] and with non-relativistic quarks [25, 26]. The comparison of the $B$ parameters with results obtained using static heavy quarks [27 30] will be addressed elsewhere [31].

Because decay constants and the corresponding matrix elements are necessary for obtaining the results of Eq. (8), we also have results for these decay constants. We find

$$
\begin{aligned}
f_{B} & =177(17)_{-22}^{+22} \mathrm{MeV} \\
f_{B_{s}} & =204(12)_{-23}^{+24} \mathrm{MeV} \\
\frac{f_{B_{s}}}{f_{B}} & =1.15(2)_{-2}^{+4} \\
\frac{f_{B}}{f_{D_{s}}} & =0.71(6)_{-5}^{+4} \\
\frac{f_{B_{s}}}{f_{D_{s}}} & =0.82(3)_{-5}^{+4}
\end{aligned}
$$

$$
\begin{aligned}
f_{D} & =210(10)_{-16}^{+17} \mathrm{MeV} \\
f_{D_{s}} & =236(8)_{-14}^{+17} \mathrm{MeV} \\
\frac{f_{D_{s}}}{f_{D}} & =1.13(2)_{-2}^{+4}
\end{aligned}
$$

where the first error is statistical and the second systematic, as discussed in Section VII. A comparison with recent quenched results 32 39] will be made in Section VII] and a discussion of unquenched results [38 41] will be undertaken

\footnotetext{
${ }^{3}$ The results of preliminary analyses on the same lattices were presented in Refs. 16 . 18 .

${ }^{4}$ We take $M_{B_{d}}=5279 \mathrm{MeV}, M_{B_{s}}=5375 \mathrm{MeV}, M_{D}=1864 \mathrm{MeV}$ and $M_{D_{s}}=1969 \mathrm{MeV}$.

${ }^{5}$ Ref. 19 gives $f_{D_{s}}=241(32) \mathrm{MeV}$ as a summary number, which is in good agreement with our determination of this quantity (see Eq. (9) below). However, recent determinations appear to yield larger values, albeit with large uncertainties: $f_{D_{s}}=285(20)(40) \mathrm{MeV}$ [20], $f_{D_{s}}=323(44)(36) \mathrm{MeV}$ 21] and $f_{D_{s}}=280(19)(44) \mathrm{MeV}$ [22].
} 
in Section VIIF. Note that results for $f_{B}$ can be combined with the measurement of the branching ratio for the rare decay $B^{+} \rightarrow \tau^{+} \nu_{\tau}$, when it becomes available, to yield a clean determination of $\left|V_{u b}\right|$. 6

While short-distance $D^{0}-\bar{D}^{0}$ mixing is highly suppressed in the standard model [48], it can be enhanced in supersymmetric extensions [49 53], above even the long-distance contributions discussed in Ref. [54]. Thus, we give the $B$ parameter and decay-constant combinations relevant for the matrix element of the left-left, $\Delta C=2$ operator, which is one of the operators that can contribute in supersymmetric extensions:

$$
\begin{gathered}
B_{D}\left(M_{D}\right)=0.82(3)_{-1}^{+4}, \quad \hat{B}_{D}^{\text {nlo }}=1.12(4)_{-1}^{+5}, \\
f_{D} \sqrt{\hat{B}_{D}^{\text {nlo }}}=222(10)_{-16}^{+20} \mathrm{MeV}, \quad \frac{f_{D}}{f_{D_{s}}} \sqrt{\hat{B}_{D}^{\text {nlo }}}=0.94(3)_{-3}^{+2},
\end{gathered}
$$

where $\hat{B}_{D}^{\text {nlo }}$ is obtained by multiplying $B_{D}\left(M_{D}\right)$ by $C_{D}\left(M_{D}\right)$, with

$$
C_{D}(\mu)=\left[\alpha_{s}(\mu)\right]^{-6 / 25}\left[1+\frac{\alpha_{s}(\mu)}{4 \pi} J_{4}\right], \quad J_{4}=\frac{6719}{3750},
$$

where the two-loop $\alpha_{s}(\mu)$ is evaluated with $\Lambda_{\frac{(4)}{\mathrm{MS}}}=350 \mathrm{MeV}$.

\section{SIMULATION DETAILS}

Our results are based on quenched, $S U(3)$ gauge configurations, calculated on a $24^{3} \times 48$ lattice at $\beta=6.2$ and a $16^{3} \times 48$ lattice at $\beta=6.0$. The configurations are generated using the hybrid over-relaxed algorithm described in Ref. [55]. The parameters of the simulations are summarized in Table [.

We describe quarks with the Sheikholeslami-Wohlert (SW) action [15] 1

$$
S_{\mathrm{F}}^{\mathrm{SW}}=S_{\mathrm{F}}^{\mathrm{W}}-i g_{0} c_{\mathrm{SW}} \frac{\kappa_{\psi}}{2} \sum_{x, \mu, \nu} \bar{\psi}(x) P_{\mu \nu}(x) \sigma_{\mu \nu} \psi(x)
$$

where $S_{\mathrm{F}}^{\mathrm{W}}$ is the standard Wilson action, $g_{0}$ the bare gauge coupling, $P_{\mu \nu}$ a lattice definition of the field strength tensor, $\kappa_{\psi}$ the appropriate quark hopping parameter and $c_{\mathrm{SW}}$, the so-called clover coefficient. Here, $\psi$ stands for both light $(q)$ and heavy $(Q)$ quarks. While with the Wilson action $\left(c_{\mathrm{Sw}}=0\right)$ spectral quantities suffer from discretization errors of $\mathcal{O}(a)$, the tree-level value, $c_{\mathrm{sw}}=1$, guarantees that these errors are reduced to $\mathcal{O}\left(\alpha_{s} a\right)$ [15, 56]. In the present paper we work with a mean-field estimate of the clover coefficient [14, 57], $c_{\mathrm{SW}}=1 / u_{0}^{3}$ with $u_{0}=\left\langle\frac{1}{3} \operatorname{Tr} U_{\mathrm{p}}\right\rangle^{\frac{1}{4}}$. Since this estimate accounts for large tadpole contributions and is closer to the nonperturbative value of the clover coefficient [58] which removes $\mathcal{O}(a)$ errors to all orders in $\alpha_{s}$, our discretization errors may be numerically smaller than for $c_{\mathrm{SW}}=1$. It is important to note, however, that in the presence of heavy quarks with masses $a m_{Q} \sim 0.3$ or more, discretization errors of $\mathcal{O}\left(\left(a m_{Q}\right)^{2}\right)$ may be comparable to those of $\mathcal{O}\left(\alpha_{s} a m_{Q}\right)$.

In order to improve matrix elements up to $\mathcal{O}\left(\alpha_{s} a\right)$, we must further "rotate" the quark fields [56]:

$$
\psi \rightarrow\left(1-\frac{a}{2}\left(z \gamma \cdot \vec{D}-(1-z) m_{\psi}\right)\right) \psi
$$

where $\vec{D}_{\mu}$ is the symmetric covariant derivative, $m_{\psi}$ the bare quark mass to be defined later and $z$ is a real parameter which can have any value between 0 and 1 . For heavy quarks, a large source of discretization errors is the mismatch in the normalization of tree-level, zero-momentum, continuum and lattice quark propagators. Eq. (13) with $z=0$ corrects this mismatch at $\mathcal{O}(a)$. Since we can compensate this mismatch completely by implementing the El-KhadraKronfeld-Mackenzie (EKM) normalization [59],

$$
\psi \rightarrow \sqrt{1+a m_{\psi}} \psi
$$

we choose the latter instead of Eq. (13).

\footnotetext{
${ }^{6}$ For recent reviews of lattice calculations of the quantities presented in Eqs. (8) and (9), please see [17, 42 47.

${ }^{7}$ Here and below $\sigma_{\mu \nu}=\frac{i}{2}\left[\gamma_{\mu}, \gamma_{\nu}\right]$, where $\gamma_{\mu}$ are the usual set of Euclidean $\gamma$-matrices with $\left\{\gamma_{\mu}, \gamma_{\nu}\right\}=2 \delta_{\mu \nu}$.
} 
At both values of the lattice spacing, we work with several values of the heavy-quark hopping parameter straddling the charm. This enables us to extrapolate our results in heavy-quark mass from the charm sector, where discretization errors appear to be only a fraction of the result, to the bottom sector, where these errors would be very large were we to perform the simulation directly with such quarks. We also consider several values of the light-quark hopping parameter around that of the strange. Then, we interpolate our results in the light-quark mass to the strange and extrapolate them to the chiral limit. The values of the hopping parameters used in our paper are given in Table II. For completeness, the masses of the corresponding light-light and heavy-light pseudoscalar mesons are also given in physical units.

To isolate the ground state more efficiently in the correlation functions that we calculate, we use fuzzed sources and/or sinks [60]. These are extended interpolation operators that have improved overlap with the ground state. Of course, operators whose matrix elements we wish to compute are kept local.

Statistical errors are estimated from a bootstrap procedure [61], which involves the creation of 1000 bootstrap samples from our set of 188 (498) configurations at $\beta=6.2(\beta=6.0)$. Correlators are fitted for each sample by minimizing $\chi^{2}$. The quoted statistical errors are obtained from the central $68 \%$ of the corresponding bootstrap distribution.

To convert our values for decay constants into physical units we need an estimate of the inverse lattice spacing. The determination of this quantity is discussed in Section $\mathrm{V}$.

\section{MATCHING AND RUNNING}

Because the lattice and the continuum treat ultraviolet modes differently, the extraction of continuum matrix elements from lattice calculations requires a matching procedure. Ideally, this matching is performed nonperturbatively. For our mean-field-improved action, however, nonperturbative matching coefficients are not available and we resort to perturbation theory instead.

The simulation is performed with the fermion action of Eq. (12) with $c_{\mathrm{SW}}=1 / u_{0}^{3}$ and the standard Wilson gauge action. However, the fully mean-field improved action would involve normalizing each occurrence of a link variable in the action by the measured value of $u_{0}$. To recover the results we would have obtained had we used the latter, we must, in interpreting the results of our simulation, use rescaled bare couplings $\bar{\alpha}_{s}=\alpha_{s} / u_{0}^{4}$ and $\bar{\kappa}_{\psi}=\kappa_{\psi} u_{0}$, with $\alpha_{s}=g_{0}^{2} /(4 \pi) . \quad c_{\mathrm{SW}}$ already has its desired rescaled value and no additional rescaling is necessary. $\bar{\alpha}_{s}$ is actually only a first guess at an improved expansion parameter and one may try to optimize this choice [14]. This issue will be elaborated on at the end of the present section and, for the moment, we will generically denote the coupling by $\alpha_{s}$.

In perturbation theory, the effect of normalizing link variables is obtained by expanding the factors of $u_{0}$ in powers of the strong coupling. At $\mathcal{O}\left(\alpha_{s}\right)$ we have 14,

$$
u_{0} \equiv\left\langle\frac{1}{3} \operatorname{Tr} U_{\mathrm{p}}\right\rangle^{\frac{1}{4}}=1+\frac{\alpha_{s}}{4 \pi} X \quad \text { with } X=-\frac{4 \pi^{2}}{3} .
$$

This means that every occurrence of $\kappa_{\psi}$ in a first order perturbative expression must be replaced by $\bar{\kappa}_{\psi}\left(1-\left(\alpha_{s} / 4 \pi\right) X\right)$. Because factors of $c_{\mathrm{SW}}$ always appear multiplied by at least one power of $\alpha_{s}$ in perturbative expressions, $c_{\mathrm{SW}}=$ $1-3\left(\alpha_{s} / 4 \pi\right) X$ can be replaced by $c_{\mathrm{sw}}=1$ in first order expressions. This is what we do to determine the central values of our results. However, in obtaining errors, we consider the variation induced by taking $c_{\mathrm{Sw}}=1 / u_{0}^{3}$.

To extract the $B$ parameters of Eq. (5) from ratios of three-point and two-point correlation functions on the lattice, we must match the mean-field improved, EKM-normalized lattice axial-vector current to its Euclidean continuum counterpart via

$$
A_{\mu}=Z_{A}\left(\alpha_{s}\right) A_{\mu}^{\text {lat }}(a)
$$

with

$$
A_{\mu}^{\text {lat }}(a) \equiv \frac{1}{a^{3}} \sqrt{1+a \bar{m}_{q}} \sqrt{1+a \bar{m}_{Q}} \sqrt{2 \bar{\kappa}_{q} 2 \bar{\kappa}_{Q}}\left(\bar{Q} \gamma_{\mu} \gamma_{5} q\right)(a)
$$


where $a \bar{m}_{q, Q}=\left(1 / \bar{\kappa}_{q, Q}-1 / \bar{\kappa}_{c r}\right) / 2$, with $\bar{\kappa}_{c r}$ the mean-field improved version of the critical hopping parameter, $\kappa_{c r}$, which is determined nonperturbatively as detailed in Section $\mathrm{V}$. Using the results of Refs. 62, 63], we find at one loop?:

$$
\begin{aligned}
Z_{A}\left(\alpha_{s}\right) & =1+\frac{\alpha_{s}}{4 \pi}\left[\frac{4}{3}\left(\Delta_{\gamma_{\mu} \gamma_{5}}+\Delta_{\Sigma_{1}}\right)-X\right] \\
& =1-\frac{\alpha_{s}}{4 \pi}\left[7.90+0.33 c_{\mathrm{SW}}-3.00 c_{\mathrm{SW}}^{2}\right],
\end{aligned}
$$

where, in the notation of Ref. [62], $\Delta_{\gamma_{\mu} \gamma_{5}}$ and $\Delta_{\Sigma_{1}}$ arise from the one-loop corrections to the vertex $\gamma_{\mu} \gamma_{5}$ and to the quark wave-function, respectively. The effect of mean-field improvement is encoded in the term proportional to $X$. Without mean-field improvement, i.e. $X=0$, the coefficient of $\alpha_{s} / 4 \pi$ would be substantially larger: 18.39 instead of 5.23 for $c_{\mathrm{SW}}=1$.

The matching of the four-quark operator $\mathcal{O}_{q}^{\Delta F=2}$ ( $F$ stands for the flavor of the heavy quark) is complicated by the fact that Wilson-type fermions break chiral symmetry explicitly, inducing mixing amongst four-quark operators of different chirality. The following five operators form a complete basis for this mixing on the lattice in the parityconserving sector:

$$
\begin{aligned}
& \mathcal{O}_{1,2}^{\text {lat }}=\gamma_{\mu} \times \gamma_{\mu} \pm \gamma_{\mu} \gamma_{5} \times \gamma_{\mu} \gamma_{5}, \\
& \mathcal{O}_{3,4}^{\text {lat }}=I \times I \pm \gamma_{5} \times \gamma_{5}, \\
& \mathcal{O}_{5}^{\text {lat }}=\sigma_{\mu \nu} \times \sigma_{\mu \nu},
\end{aligned}
$$

where a sum over Lorentz indices is implicit and where $\Gamma \times \Gamma$ stands for $\left(1 / a^{6}\right)\left(1+a \bar{m}_{q}\right)\left(1+a \bar{m}_{Q}\right)\left(2 \bar{\kappa}_{q} 2 \bar{\kappa}_{Q}\right)$ $(\bar{Q} \Gamma q)(\bar{Q} \Gamma q)$. The parity even component of $\mathcal{O}_{q}^{\Delta F=2}$ corresponds to the continuum equivalent of $\mathcal{O}_{1}^{\text {lat }}$ so that, at one loop:

$$
\mathcal{O}_{q}^{\Delta F=2}(\mu) \longrightarrow Z_{11}\left(\alpha_{s}, a \mu\right)\left(\mathcal{O}_{1}^{\text {lat }}(a)+\sum_{i=2}^{5} Z_{1 i}\left(\alpha_{s}\right) \mathcal{O}_{i}^{\text {lat }}(a)\right)
$$

$Z_{11}$ has a logarithmic dependence on $a \mu$, where $\mu$ is the scale at which the continuum operator is renormalized, while $Z_{1 i}, i=2, \cdots, 5$, remains finite as $a$ vanishes. Mean-field improving the dimensional reduction (DRED) results of Refs. 62 64 and using the matching between DRED and NDR- $\overline{\mathrm{MS}}$ given in Ref. [65], we find that one-loop matching to the NDR- $\overline{\mathrm{MS}}$ scheme is given by:

$$
\begin{aligned}
Z_{11}\left(\alpha_{s}, a \mu\right)=1 & +\frac{\alpha_{s}}{4 \pi}\left[-4 \ln (a \mu)+\frac{1}{3}\left\{5\left(\Delta_{\gamma_{\mu}}+\Delta_{\gamma_{\mu} \gamma_{5}}\right)\right.\right. \\
& \left.\left.-\left(\Delta_{I}+\Delta_{\gamma_{5}}\right)+8 \Delta_{\Sigma_{1}}\right\}-2-2 X\right] \\
= & 1-\frac{\alpha_{s}}{4 \pi}\left[4 \ln (a \mu)+24.52-9.33 c_{\mathrm{SW}}-4.88 c_{\mathrm{SW}}^{2}\right], \\
Z_{12}\left(\alpha_{s}\right)= & -\frac{11}{12} \frac{\alpha_{s}}{4 \pi}\left[\Delta_{I}-\Delta_{\gamma_{5}}\right]=-\frac{\alpha_{s}}{4 \pi}\left[8.84-9.15 c_{\mathrm{SW}}+3.13 c_{\mathrm{SW}}^{2}\right], \\
Z_{13}\left(\alpha_{s}\right)= & -\frac{4}{11} Z_{12}\left(\alpha_{s}\right), Z_{14}\left(\alpha_{s}\right)=\frac{2}{11} Z_{12}\left(\alpha_{s}\right), Z_{15}\left(\alpha_{s}\right)=\frac{2}{11} Z_{12}\left(\alpha_{s}\right),
\end{aligned}
$$

where the $\Delta_{\Gamma}, \Gamma=\gamma_{\mu}, \gamma_{\mu} \gamma_{5}, I$ and $\gamma_{5}$ arise from the one-loop corrections to the bilinear vertices associated with $\Gamma$. 9

For the numerical evaluation of the renormalization constants, we choose to work with the $\overline{\mathrm{MS}}$ coupling, $\alpha_{\overline{\mathrm{MS}}}$, obtained via

$$
\alpha_{\overline{\mathrm{MS}}}(3.41 / a)=\alpha_{V}\left(\mathrm{e}^{5 / 6} 3.41 / a\right)\left(1+2 \frac{\alpha_{V}}{\pi}\right)
$$

\footnotetext{
${ }^{8}$ In practice, we use [63 where results are given for arbitrary $c_{\mathrm{SW}}$ and where loop integrals are calculated to higher numerical accuracy.

${ }^{9}$ Here again, we use the more precise and general $c_{\mathrm{SW}}$ results of Ref. 63] for $\Delta_{\Gamma}$.
} 
where $\alpha_{V}$ is the coupling defined from the heavy-quark potential. The latter is obtained from our simulations by solving [14]

$$
\ln \left(u_{0}\right)=\frac{\pi}{3} \alpha_{V}(3.41 / a)\left(1-1.185 \alpha_{V}\right) .
$$

Values of $\alpha_{\overline{\mathrm{MS}}}$ for scales different from 3.41/a are obtained by solving the two-loop running equation numerically, with $n_{f}=0$. Both $\alpha_{\overline{\mathrm{MS}}}$ and $\alpha_{V}$ have been shown to lead to expansions that are much more convergent than those in terms of the bare lattice coupling [14]. For completeness, we give values of $\alpha_{\overline{\mathrm{MS}}}(\mu)$ for a collection of $\mu$ in Table III].

Having chosen the coupling, we must fix the scale, $q^{*}$, at which it is evaluated. We take $2 / a$ as a central value but allow $q^{*}$ to vary from $1 / a$ to $\pi / a$ to estimate the uncertainty associated with this choice. This range for $q^{*}$ covers typical, ultraviolet lattice scales.

As it is convenient for the heavy-quark extrapolations and does not generate large logarithms, we match the lattice results for the $B$ parameters at $\mu=M_{P_{l}}$, where $M_{P_{l}}$ is the mass of the heavy-light meson composed of a heavy antiquark $\bar{Q}$ and a massless quark, $l=d$ or $u$. As we will see in Section V1, the heavy-quark extrapolation yields $B_{B_{q}}\left(M_{B_{d}}\right)$. Values for the renormalization-group-invariant and scheme-independent $B$ parameters are then simply obtained from Eqs. (7) and (3): $\hat{B}_{B_{q}}^{\text {nlo }}=C_{B}\left(M_{B_{d}}\right) B_{B_{q}}\left(M_{B_{d}}\right)$. Values for $B_{B_{q}}(\mu)$, with $\mu \neq M_{B_{d}}$, can also be obtained straightforwardly through: $B_{B_{q}}(\mu)=\left[C_{B}\left(M_{B_{d}}\right) / C_{B}(\mu)\right] B_{B_{q}}\left(M_{B_{d}}\right)$, with or without an expansion in $\alpha_{s}$ of the term proportional to $J_{5}$ in Eq. (3)

Since we match the matrix elements and $B$ parameters defined in Eq. (5) at one loop, we may choose to expand combinations of renormalization constants such as $Z_{11} / Z_{A}^{2}$ or $Z_{11} Z_{1 i}, i=2, \cdots, 5$, to order $\alpha_{s}$. In the present paper, all central values are obtained without expansion. Nevertheless, we have checked that expanding these combinations makes negligible differences in the final results. This is because the one-loop corrections to the renormalization constants are small, especially after mean-field improvement.

\section{CORRELATION FUNCTIONS}

To determine $\Delta F=2$ matrix elements and their $B$ parameters, as well as decay constants, we compute the following two- and three-point functions:

$$
\begin{aligned}
C_{P P}^{F F}\left(t_{x}\right) & =\sum_{\vec{x}}\left\langle P_{F}^{\dagger}(x) P_{F}(0)\right\rangle, \\
C_{A P}^{L F}\left(t_{x}\right) & =\sum_{\vec{x}}\left\langle P_{F}^{\dagger}(x) A_{0}^{\mathrm{lat}}(0)\right\rangle, \\
C_{\mathcal{O}}^{F L F}\left(t_{x}, t_{y}\right) & =\sum_{\vec{x}, \vec{y}}\left\langle P_{F}^{\dagger}(y) \mathcal{O}(0) P_{F}^{\dagger}(x)\right\rangle,
\end{aligned}
$$

as well as $C_{P A}^{F L}\left(t_{x}\right)$ and $C_{P P}^{F L}\left(t_{x}\right)$. Here, the subscripts $F$ on operators indicate that they are fuzzed [60], while operators with no $F$ are local. For the correlation functions, the superscripts $F$ and $L$ indicate which of their operators are fuzzed $(F)$ or local $(L)$. In Eq. (24), $A_{0}^{\text {lat }}$ is the time component of the EKM-normalized axial-vector current defined in Eq. (17), $P_{F}$ is a fuzzed [60] version of the pseudoscalar density $\bar{Q} \gamma_{5} q$, and $\mathcal{O}$ stands for any of the four-fermion operators in Eqs. (19) and (20). In the present section, we set the lattice spacing $a=1$ and omit light-quark indices on heavy-light quantities, for notational simplicity.

At large Euclidean time separations, the three-point correlation function $C_{\mathcal{O}}^{F L F}\left(t_{x}, t_{y}\right)$ has the asymptotic behavior 19

$$
C_{\mathcal{O}}^{F L F}\left(t_{x}, t_{y}\right) \rightarrow \frac{1}{4 M_{P}^{2}}\left\langle 0\left|P_{F}^{\dagger}\right| \bar{P}\right\rangle\langle\bar{P}|\mathcal{O}| P\rangle\left\langle P\left|P_{F}^{\dagger}\right| 0\right\rangle \exp \left[-M_{P}\left(T-t_{x}+t_{y}\right)\right]
$$

\footnotetext{
${ }^{10}$ On our periodic lattices this corresponds to $a t_{y}$ and $a\left(T-t_{x}\right)$ much greater than 1 but small enough so that the desired time ordering dominates. Here, $a T=48$ is the time extent of our lattices. We consider times $t_{x}$ and $t_{y}$ such that $T / 2<t_{x}<T$ and $0<t_{y}<T / 2$.
} 
where $M_{P}$ is the mass of the pseudoscalar $P$. Therefore, fits to the ratios of correlation functions:

$$
\begin{aligned}
\frac{C_{\mathcal{O} \Delta F=2}^{F L F}\left(t_{x}, t_{y}\right)}{C_{A P}^{L F}\left(t_{x}\right) C_{A P}^{L F}\left(t_{y}\right)} \longrightarrow \frac{8}{3} Z_{A}^{2} B_{P}, \\
\frac{C_{\mathcal{O}_{q}^{\Delta F=2}}^{F L F}\left(t_{x}, t_{y}\right)}{C_{P P}^{F F}\left(t_{x}\right) C_{P P}^{F F}\left(t_{y}\right)} \longrightarrow \frac{\left\langle\bar{P}\left|\mathcal{O}_{q}^{\Delta F=2}\right| P\right\rangle}{\left\langle 0\left|P_{F}\right| P\right\rangle\left\langle\bar{P}\left|P_{F}\right| 0\right\rangle},
\end{aligned}
$$

where $B_{P}$ is the $B$ parameter corresponding to the heavy-light meson $P$, yield the desired quantities up to renormalization constants that we determine perturbatively (see Section IIII) and factors of $M_{P}$ and of the type, $\left\langle 0\left|P_{F}\right| P\right\rangle$, that we determine from fits to the two-point functions $C_{P P}^{F L}\left(t_{x}\right)$ and $C_{P P}^{F F}\left(t_{x}\right)$, respectively. Note that in Eqs. (25) and (27), all pseudoscalar meson states have vanishing three momentum. An example of a plateau for the ratio of Eq. (26) is shown in Figure 11.

To determine the decay constants, we consider

$$
\frac{C_{P A}^{F L}\left(t_{x}\right)}{C_{P P}^{F F}\left(t_{x}\right)} \longrightarrow \frac{M_{P} f_{P}}{Z_{A}\left\langle 0\left|P_{F}^{\dagger}\right| \bar{P}\right\rangle} \tanh \left[M_{P}\left(T / 2-t_{x}\right)\right]
$$

In order to investigate the dependence of the matrix elements and $B$ parameters on heavy- and light-quark mass, we need the heavy-light and the light-light pseudoscalar meson masses, and the light-light decay constants, which we use to set the scale. As already stated, we obtain the heavy-light pseudoscalar meson mass, $M_{P}$, from a fit to the large time behavior of $C_{P P}^{F L}\left(t_{x}\right)$. Similarly, we get the light-light pseudoscalar meson mass, $m_{p}$, from a study of the two-point function $C_{p p}^{F L}\left(t_{x}\right)$ where $p=\bar{q}_{1} \gamma_{5} q_{2}$ is the pseudoscalar bilinear made from the two light quarks $q_{1}$ and $q_{2}$. Finally, the light-light pseudoscalar decay constant is obtained from a fit to the ratio $C_{p a}^{F L}\left(t_{x}\right) / C_{p p}^{F F}\left(t_{x}\right)$, where $a_{0}$ is the EKM-normalized version of $\bar{q}_{1} \gamma_{0} \gamma_{5} q_{2}$, much in the same way the heavy-light decay constant is obtained.

The time ranges over which the various correlation functions and ratios are fitted are given in Table IV.

\section{LIGHT-QUARK-MASS EXTRAPOLATIONS AND DETERMINATION OF THE LATTICE SPACING}

Results for physical $B_{d}$ and $B_{s}$ mesons require investigations of the dependence of the lattice measurements on light- and heavy-quark masses. We begin by the light-quark-mass extrapolations and interpolations. To obtain $\kappa_{c r}$, the critical value of the quark hopping parameter, we study the behavior of the light-light pseudoscalar meson mass, $m_{p}$, as a function of $\kappa_{1}$ and $\kappa_{2}$, the hopping parameters of the light quarks which compose it. We assume that it obeys the partially conserved axial-current (PCAC) relation

$$
\left(a m_{p}\right)^{2}\left(\kappa_{1}, \kappa_{2}\right)=\beta_{m}\left(a \tilde{m}_{1}+a \tilde{m}_{2}\right),
$$

where $\tilde{m}_{i}$ is the $O(a)$-improved quark mass, given by

$$
\tilde{m}_{i}=m_{i}\left(1+b_{m} a m_{i}\right)
$$

with $a m_{i}=1 /\left(2 \kappa_{i}\right)-1 /\left(2 \kappa_{c r}\right)$ [66]. At tree level, which is sufficient with our mean-field-improved action, $b_{m}=-1 / 2$. $\kappa_{c r}$ is then obtained by fitting the function of Eq. (29) to our results for $\left(a m_{p}\right)^{2}$, with $\beta_{m}$ and $\kappa_{c r}$ as fit parameters.

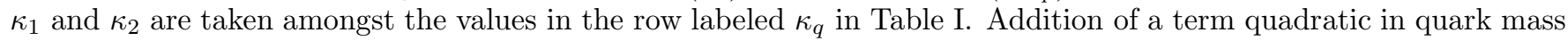
in Eq. (29) makes very little difference to the central value for $\kappa_{c r}$ and this variation has a negligible effect on the light-quark-mass extrapolations of the matrix elements of interest. The linear extrapolation of Eq. (29) is shown in Figure 2.

For $m_{p}$ and all the quantities we study here, in addition to higher-order polynomial corrections in light-quark mass, chiral perturbation theory also predicts the presence of chiral logarithms. These logarithms, however, are difficult to isolate numerically and are modified by the quenched approximation 67 69]. Thus, we perform polynomial

\footnotetext{
${ }^{11} C_{P P}^{F L}$ gives a particularly good signal for $M_{P}$.
} 
interpolations and extrapolations from our intermediate values of light-quark mass, only considering in most cases the leading, non-trivial dependence on light-quark mass.

Next we determine the lattice scale $a^{-1}$ and the bare strange-quark mass, $a \tilde{m}_{s}$, in lattice units. We obtain both quantities simultaneously from the kaon's decay constant, $f_{K}$, and its mass, $m_{K}$. As long as the mass dependence of $f_{p}$, the decay constant of a light-light pseudoscalar meson, can be described by a function of $m_{p}^{2}$ only, Eq. (29) implies that both quantities depend only on the sum of quark masses and that only an interpolation in these masses, not an extrapolation, is needed to obtain $a f_{K}$. The use of $f_{K}$, instead of, for instance, $m_{K^{*}}$, is prompted by the fact that the only quantities we report on here which depend strongly (i.e. not logarithmically) on $a^{-1}$ are the heavy-light decay constants. Our procedure has the added benefit that it is also applicable in unquenched simulations ${ }^{12}$. Thus, we fit our results for $a f_{p} / Z_{A}$ to:

$$
Z_{A}\left(\frac{a f_{p}}{Z_{A}}\right)=\alpha_{f}+\beta_{f}\left(a m_{p}\right)^{2}+\gamma_{f}\left(a m_{p}\right)^{4}+\cdots
$$

We find that this parametrization describes our results well. Assuming that the lattice spacing is fixed with $f_{K}$ (or equivalently $m_{K}$ ), we solve Eq. (31) for $a f_{p}$ (or $a m_{p}$ ), at the point specified by the physical ratio $m_{K} / f_{K}$, with $f_{K}=159.8 \mathrm{MeV}$ and $m_{K}=493.7 \mathrm{MeV} . a \tilde{m}_{s}$ is then obtained from the resulting $a m_{K}$, using our earlier fit to Eq. (29), and the lattice spacing from the resulting $a f_{K}$ (or $\left.a m_{K}\right)$. The fits at our two values of the coupling are shown in Figure 3. Because of the slight curvature, we favor the quadratic fits. These fits also give a value of $f_{K} / f_{\pi}$ which is closer to the experimental result of 1.22: 1.16(3) (1.19(2)) for quadratic fits instead of 1.131(10) $(1.147(7))$ for linear fits, at $\beta=6.2(6.0)$. In any case, linear and quadratic fits give nearly identical results for the scale and $a \tilde{m}_{s}$. The values of $Z_{A}$ used are those obtained from Eq. (18) with $\alpha_{s}=\alpha_{\overline{\mathrm{MS}}}(2 / a)$. Systematic uncertainties in the determination of $a^{-1}$ and $a \tilde{m}_{s}$ will be addressed when we discuss the uncertainties on our $B$-physics results in Section VII. A summary of the results for $a^{-1}, \kappa_{c r}$ and $a \tilde{m}_{s}$ used below is given in Table $\mathrm{V}$.

Having determined the strange quark mass and critical hopping parameter, we interpolate and extrapolate our various heavy-light matrix elements in light-quark mass to these values. We assume that the up and the down quarks are massless. This is an excellent approximation for the quantities we study. As mentioned above, we perform polynomial interpolations and extrapolations from our values of light-quark mass. Thus, we fit all quantities of interest, $Y$, in lattice units, to the functional form:

$$
Y\left(\kappa_{Q}, \kappa_{q}\right)=\alpha_{Y}+\beta_{Y} a \tilde{m}_{q}+\cdots .
$$

In Figures 4, 5, 6 and 7 we exhibit the light-quark-mass dependence and the corresponding fits to Eq. (32) for the quantities $1 /\left(a M_{P_{q}}\right), B_{P_{q}}, a^{4}\left\langle\bar{P}_{q}\left|O_{q}^{\Delta F=2}\right| P_{q}\right\rangle$ and $a f_{P_{q}} / Z_{A}$, for all values of heavy-quark mass at $\beta=6.0$ and 6.2 . For clarity of presentation, the $B$ parameters and $a^{4}\left\langle\bar{P}_{q}\left|O_{q}^{\Delta F=2}\right| P_{q}\right\rangle$ are renormalized at a common scale of $5 \mathrm{GeV}{ }^{13}$. In all cases, the light-quark-mass dependence is mild and, to good accuracy, linear. However, we do observe that the matrix elements of $O_{q}^{\Delta F=2}$ have a stronger dependence on light-quark mass than the other quantities.

\section{HEAVY-QUARK-MASS EXTRAPOLATIONS}

The second extrapolation we have to perform is in heavy-quark mass. We use heavy quark effective theory (HQET) as a guide, with $M_{P_{l}}$ as a measure of the heavy-quark mass everywhere except for kinematical dependencies, where the appropriate meson mass is used. As before, $l$ stands for either a $d$ or a $u$ quark. Other choices for the heavy-quark scale, such as $M_{P_{s}}$, for instance, make very little difference to the final results. We match our QCD results onto HQET at the heavy-quark scale $M_{P_{l}}$, and cancel the leading logarithmic dependence on $M_{P_{l}}$ by including terms of the form $\left[\alpha_{s}\left(M_{P_{l}}\right)\right]^{-\gamma_{0}^{\mathrm{HQET}} / 2 \beta_{0}}$, where $\gamma_{0}^{\mathrm{HQET}}$ are the relevant, one-loop anomalous dimensions in HQET, and $\beta_{0}$ is the one-loop $\beta$-function coefficient. $\gamma_{0}^{\mathrm{HQET}}=-4$ and -8 for the decay constant and four-quark matrix element, respectively [70, 71 . Thus, we define

\footnotetext{
${ }^{12}$ The $K^{*}$ is not a stable particle once light-quark loops are allowed.

${ }^{13}$ These values of $B_{P_{q}}$ and $a^{4}\left\langle\bar{P}_{q}\left|O_{q}^{\Delta F=2}\right| P_{q}\right\rangle$ are obtained by matching the lattice results onto the NDR-MS scheme at the scale $2 / a$, then running to $5 \mathrm{GeV}$ in the $\overline{\mathrm{MS}}$ scheme at the two-loop level with $n_{f}=0$ and the coupling constant described in Section III.
} 


$$
\Phi_{f}^{q}\left(M_{P_{l}}\right) \equiv \frac{a f_{P_{q}}}{Z_{A}} \sqrt{a M_{P_{q}}} \times\left[\alpha_{s}\left(M_{P_{l}}\right)\right]^{2 / \beta_{0}}
$$

for the decay constants,

$$
\Phi_{B}^{q}\left(M_{P_{l}}\right) \equiv B_{P_{q}}\left(M_{P_{l}}\right) \times\left[\alpha_{s}\left(M_{P_{l}}\right)\right]^{0 / \beta_{0}},
$$

for the $B$ parameters, and

$$
\Phi_{\Delta F=2}^{q}\left(M_{P_{l}}\right) \equiv a^{4}\left\langle\bar{P}_{q}\left|\mathcal{O}_{q}^{\Delta F=2}\left(M_{P_{l}}\right)\right| P_{q}\right\rangle \frac{1}{a M_{P_{q}}} \times\left[\alpha_{s}\left(M_{P_{l}}\right)\right]^{4 / \beta_{0}},
$$

for the $\Delta F=2$ matrix element. Similar scaling functions are defined for $S U(3)$-breaking ratios, in which the leading logarithmic dependence on heavy-quark mass cancels. In Eqs. (33), (34) and (35), we evaluate $\alpha_{s}(M)$ through $2 \pi /\left(\beta_{0} \log \left(M / \Lambda_{\mathrm{QCD}}\right)\right)$ with $\Lambda_{\mathrm{QCD}}=100 \mathrm{MeV}$ and $\beta_{0}=11-(2 / 3) n_{f}$ with $n_{f}=0$, since we are working in the quenched approximation. This one-loop coupling approximates the lattice couplings defined through Eqs. (22) and (23) rather well, for the values of $M$ required here. In fact, final results depend weakly on the value $\Lambda_{\mathrm{QCD}}$ used in the heavy-quark extrapolations (see also Figure 8 and discussion below).

For $X\left(M_{P_{l}}\right)=\Phi_{\Delta F=2}^{q}\left(M_{P_{l}}\right), \Phi_{B}^{q}\left(M_{P_{l}}\right), \Phi_{f}^{q}\left(M_{P_{l}}\right)$ and the corresponding scaling functions for $S U(3)$-breaking ratios, we use the HQET-inspired relation

$$
X\left(M_{P_{l}}\right)=A_{X}\left[1+B_{X}\left(\frac{1}{a M_{P_{l}}}\right)+C_{X}\left(\frac{1}{a M_{P_{l}}}\right)^{2}+\cdots\right]
$$

to investigate the heavy-quark-mass scaling of these quantities. The leading logarithms make little difference in the extrapolation, as shown in Figure 8 where we plot the extrapolation of the $\Delta F=2$ matrix element, which has the strongest logarithmic dependence amongst the quantities we study, with and without these logarithms.

In Figures 9 and 10 we display our results for $\Phi_{B}^{q}\left(M_{P_{l}}\right)$ and $\Phi_{\Delta F=2}^{q}\left(M_{P_{l}}\right)$, constructed from $B_{P_{q}}\left(M_{P_{l}}\right)$ and $\left\langle\bar{P}_{q}\left|\mathcal{O}_{q}^{\Delta F=2}\left(M_{P_{l}}\right)\right| P_{q}\right\rangle$ renormalized at $M_{P_{l}}$ in the NDR-MS scheme, as functions of inverse heavy-meson mass at $\beta=6.0$ and 6.2 and for $q=s$ and $l$. In Figure 11, we plot $\Phi_{f}^{q}\left(M_{P_{l}}\right)$ versus $1 /\left(a M_{P_{l}}\right)$. Finally, in Figures 12, 13 and 14 we plot the extrapolations of the corresponding $S U(3)$-breaking ratios. The fit parameters of the heavy-quark-mass scaling of the various quantities studied are summarized in Table $\mathrm{VI}$. While the heavy-quark-mass dependence of most quantities is mild, that of $\Phi_{f}^{q}\left(M_{P_{l}}\right)$ and especially $\Phi_{\Delta F=2}^{q}\left(M_{P_{l}}\right)$ is quite severe.

We extrapolate $S U(3)$-breaking ratios directly in heavy-quark mass because the mass dependence cancels partially between numerator and denominator, making the extrapolation less pronounced and, thus, more reliable. This is especially visible for $f_{P_{s}} / f_{P_{l}}$ (Figure 14), where the extrapolation of the ratio yields much smaller uncertainties than the ratio of the extrapolations. In all cases, the extrapolation of the ratio is in excellent agreement with the ratio of extrapolations. It is interesting to note, also, that the heavy-quark-mass dependence of $f_{P_{s}} / f_{P_{l}}$ appears to be the same as that of $\sqrt{M_{P_{l}} / M_{P_{s}}}$.

\section{SYSTEMATIC UNCERTAINTIES}

Our main results at the two values of lattice spacing are summarized in Tables VII, VIII and IX. In these tables, the first error on each quantity is statistical. The remaining uncertainties are systematic and we discuss them now.

\section{A. Discretization errors}

Ideally, one would extrapolate all computed quantities to the continuum limit, where discretization errors vanish. With two values of the lattice spacing, however, this is not possible. We must therefore use the information that we have to estimate the uncertainty associated with residual discretization effects.

In Table VII, results for the decay constants display some dependence on lattice spacing. This suggests that discretization errors for these quantities may be important. The leading discretization errors with the mean-fieldimproved SW action are formally of $\mathcal{O}\left(\alpha_{s} a\right)$, as they are for the tree-level improved SW action. Subleading errors 
begin at $\mathcal{O}\left(\alpha_{s}^{2} a\right){ }^{14}$ and $\mathcal{O}\left(a^{2}\right)$. To estimate these leading and subleading errors, we consider the following variations in our procedure. $\mathcal{O}\left(\alpha_{s} a\right)$ improvement of the axial current requires one to include the effect of the $a \partial_{\mu} P$ counterterm through the replacement ( $P$ is the pseudoscalar density)

$$
A_{\mu} \rightarrow A_{\mu}+c_{A} a \partial_{\mu} P
$$

as well as to rescale the quark fields as

$$
\psi \rightarrow\left(1+\frac{b_{A}}{2} a m_{\psi}\right) \psi
$$

with both $c_{A}$ and $b_{A}$ evaluated at one loop [66, 72]. From a comparison of results obtained with $c_{A}$ and $b_{A}$ set to their tree-level values $\left(c_{A}=0\right.$ and $\left.b_{A}=1\right)$ to those obtained with $c_{A}$ and $b_{A}$ evaluated at one loop, we can estimate the effect of $\mathcal{O}\left(\alpha_{s} a\right)$ discretization errors. We do not use the one-loop results as central values for the decay constants to be consistent with our determination of the $B$ parameters. Indeed, $\mathcal{O}\left(\alpha_{s} a\right)$ improvement of the four-quark operators would require one to consider the mixing of these operators with operators of dimension seven, which is beyond the scope of the present paper.

As already mentioned, to subtract higher-order discretization effects, we use the EKM normalization of Eq. (14). Thus we define the one-loop variation in the normalization of the quark fields through

$$
\psi \rightarrow \frac{\sqrt{1+a \bar{m}_{\psi}}}{1+a \bar{m}_{\psi} / 2}\left(1+\frac{b_{A}^{1-\text { loop }}}{2} a \bar{m}_{\psi}\right) \psi
$$

where, using the results of $\sqrt{73}$, we find $b_{A}^{1-\text { loop }}=1+\left(\alpha_{s} / 4 \pi\right)[24.03+X]=1+10.87\left(\alpha_{s} / 4 \pi\right)$ for $c_{\mathrm{Sw}}=1 . \quad c_{A}^{1-\text { loop }}$ is given by $-1.20\left(\alpha_{s} / 4 \pi\right)$ [73]. We find that the replacements of Eqs. (37) and (39) have opposite effects. The former lowers the decay constants while the latter increases their values. For the one-loop values of $c_{A}$ and $b_{A}$, the cancellation is rather good and the resulting one-loop versus tree-level variation is certainly an underestimate of the residual discretization errors proportional to $a$. To get a more realistic estimate, we consider the variation brought about by each replacement separately. These variations are shown as the second error on the decay constants and their $S U(3)$-breaking ratios, $f_{D_{s}} / f_{D}$ and $f_{B_{s}} / f_{B}$. We take the largest of the two to be a measure of residual discretization errors proportional to $a$. 15

To get a handle on errors proportional to higher powers of $a$, we consider the result of using the tree-level, quarkfield normalization of Eq. (38) with $b_{A}=1$, instead of the EKM normalization of Eq. (14). These two normalizations differ at $\mathcal{O}\left(a^{2}\right)$ and we take the resulting variation to be a measure of these additional discretization errors. This variation is shown as the third error on the decay constants and their $S U(3)$-breaking ratios, $f_{D_{s}} / f_{D}$ and $f_{B_{s}} / f_{B}$.

The symmetric discretization error that enters the systematic error in the final results of Eq. (9) is obtained by combining in quadrature our estimates of the residual discretization uncertainties proportional to $a$ and of the uncertainties proportional to higher powers of $a$. While these two uncertainties are comparable at $\beta=6.2$, the latter are significantly larger at $\beta=6.0$ in the $b$-quark sector.

A similar estimate of discretization errors can be carried out for the four-quark matrix elements and their $B$ parameters. However, as we have already mentioned a full quantification of $\mathcal{O}\left(\alpha_{s} a\right)$ effects for these quantities is beyond the scope of this paper. In fact, many discretization effects, such as those associated with the normalization of quark fields, cancel or partially cancel in the ratios of matrix elements used to define the $B$ parameters and $S U(3)$ breaking ratios. Furthermore, in Table VIII, results for $B$ parameters and $S U(3)$-breaking ratios exhibit very little lattice-spacing dependence, supporting the idea that discretization errors for these quantities are small. Thus, we assume that their statistical uncertainties encompass possible residual discretization errors. For the quantities in Table IX, however, which are obtained using the decay constants, we take into account the discretization errors on these constants.

\footnotetext{
${ }^{14} \mathcal{O}\left(\alpha_{s}^{2} a\right)$ errors, as well as all errors proportional to $a$, are absent in non-perturbatively $\mathcal{O}(a)$-improved calculations.

${ }^{15}$ The largest variation is the one of Eq. (39).
} 


\section{B. Matching uncertainties}

As already indicated in Section III, to estimate the systematic errors arising from the perturbative matching of the various quantities we compute, we vary the scale, $q^{*}$, at which $\alpha_{\overline{\mathrm{MS}}}$ is evaluated, in the range between $1 / a$ and $\pi / a$, and compare this with the result obtained for $q^{*}=2 / a$. We also consider the variation coming from computing $Z_{11}\left(M_{P_{l}}\right), Z_{1 i}$ and $Z_{A}$ with the constant $c_{\mathrm{Sw}}$ set to its mean-field-improved value instead of 1 , keeping $q^{*}=2 / a$ fixed. $S U(3)$-breaking ratios of decay constants are not affected by these variations while those of the $B$ parameters are not significantly so. These variations are reflected in the fourth error in Tables VII, VIII and IX]

\section{Heavy-quark-mass extrapolations}

As shown in Figure 11, the decay constants have a pronounced extrapolation in heavy-quark mass, and the term quadratic in $1 /\left(a M_{P_{l}}\right)$ on the right-habd side of Eq. (36) contributes significantly. To quantify the systematic error associated with this extrapolation-the fifth error on the decay constants-we perform a fit of the heaviest three points in Figure 11 to the right-hand side of Eq. (36), without the quadratic term. For the $S U(3)$-breaking ratios of decay constants, we perform a constant fit to these same three points. These uncertainties are propagated to the results of Table IX.

Figure 9 indicates that the heavy-quark-mass dependence of the $B$ parameters and their $S U(3)$ breaking ratio is mild and to very good approximation linear. We have verified that a linear fit to the three heaviest points gives results that are well within the errors bars of the fit to all five points at $\beta=6.0$. We assume that the same would be true at $\beta=6.2$ if we also had five heavy quarks, as there is no evidence for curvature on the three points that we have.

The $\Delta F=2$ matrix elements have a very pronounced dependence on heavy-quark mass, as seen in Figure 10. Since we are not reporting results for the four-quark matrix elements themselves, we do not quantify the systematic errors associated with their determination.

One may worry that we have only three heavy quarks at $\beta=6.2$ in our calculation of $\Delta F=2$ matrix elements. However, as we have just seen, the heavy-quark scaling of the $B$ parameters and their $S U(3)$-breaking ratio is mild and displays no evidence for curvature. This is confirmed by the behavior at $\beta=6.0$ where we have five heavy quarks. Thus, we believe that our results for $B$ parameters and the derived quantities of Table IX at $\beta=6.2$ are reliable. The situation is certainly not as favorable for the $\Delta F=2$ matrix elements themselves. For those, there is evidence for curvature and our three points at $\beta=6.2$ can be thought to yield only a rough estimate. Thus, we do not attempt to give a final result for these matrix elements from this procedure. In fact, this strong mass dependence is one of the problems that makes a reliable determination of $r_{s d}$, from the ratio of individually calculated $\left\langle\bar{B}_{q}\left|\mathcal{O}_{q}^{\Delta B=2}\right| B_{q}\right\rangle$, $q=s, d$, difficult. The extrapolation of $r_{s d}$ itself, on the other hand, is much milder and the curvature is much reduced. Thus, we extrapolate it linearly and verify, at $\beta=6.0$ where we have enough points, that the result of a quadratic fit, $r_{s d}^{\text {direct }}=1.50(17)$, is entirely compatible. ${ }^{16}$ In any event, the final value of $r_{s d}$ that we quote is that given by the "indirect" method, where none of this is a problem.

Another concern may be that our lightest heavy quark is too light to be in the heavy quark scaling regime. However, in the extrapolations used to obtain the results of Eqs. (8) and (9), the points corresponding to this quark are consistent with the smooth curves determined by the other points. Furthermore, our heaviest quarks are as massive as those in other relativistic calculations (see for instance Ref. 24]). Thus, we are not distorting the heavy-quark extrapolations by including these lighter points, nor are we missing information on the heavier-quark end. Finally, as described above, we include in our errors, the variation obtained by ignoring our lightest two points, where appropriate.

Ideally, one would have continuum extrapolations of results such as ours and of the same quantities computed in the static limit (corresponding to an infinite-mass heavy quark). Results for the $b$ would then be obtained by an interpolation in heavy-quark mass instead of by extrapolation, as they are here. We leave such studies for the future.

\footnotetext{
${ }^{16}$ To estimate the heavy-quark extrapolation error at $\beta=6.0$, we consider the variation due to the removal of the lightest two points from the linear extrapolation of Fig. 13 .
} 


\section{Uncertainties in the determination of the lattice spacing}

In quenched calculations, the value of the lattice spacing varies significantly with the quantity used to set the scale. This variation may be due, in part, to quenching effects, as well as any other systematic uncertainty that affects the quantity used to set the scale. In this paper, we determine the lattice spacing from $f_{K}$ and $m_{K}$, as described in Section $\mathrm{V}$. We then vary the inverse lattice spacing, $a^{-1}$, by $\pm 7 \%$. This range covers the variations observed in the determination of the scale from gluonic or light-hadron spectral quantities, and with the same action and parameters as we use 74 177, as well as the variation due to the uncertainty in the perturbative determination of $Z_{A}$.

Uncertainties in the lattice spacing will obviously affect the determination of the decay constants and the dimensionful quantities derived from them. They will also slightly change the length of the heavy-quark-mass extrapolations (Figures 11, 9 and 10). Furthermore, they induce a variation of order $\pm 15 \%$ in $a \tilde{m}_{s}$, which we obtain from the mass of the kaon, and therefore affect all quantities that depend on this mass.

In practice, we find that the variation of the lattice spacing discussed above does not induce a significant change in the $B$ parameters. However, it does affect all the decay constants and the corresponding $S U(3)$-breaking ratios as well as the quantities in Table IX] which are obtained from these constants. All of these observations are reflected in the sixth error on the quantities in Tables VII, VIII and IX.

\section{E. Determination of the light-quark masses}

Another source of systematic error is the uncertainty in the determination of the light-quark masses. In the previous section we saw that changing the lattice spacing, while keeping fixed the physical quantity used to set the strangequark mass, induced approximatively a $\pm 15 \%$ variation in $a \tilde{m}_{s}$. One can also imagine doing the reverse, i.e. holding the lattice spacing fixed while varying the observable used to set the strange-quark mass. For instance, we could have used the $K^{*}$ or $\phi$ meson masses to fix $a \tilde{m}_{s}$, instead of $m_{K}$. Let us denote the resulting values of the strange quark mass by $a \tilde{m}_{s}\left(m_{K^{*}}\right)$, etc. Due to quenching and other systematic effects, the values obtained may differ.

To estimate these differences, we turn to the literature. In Ref. [75], where the determination of quark masses with different fermionic actions is reviewed thoroughly, Bhattacharya and Gupta conclude that for mean-field-improved SW actions such as ours, $a \tilde{m}_{s}\left(m_{\phi}\right) / a \tilde{m}_{s}\left(m_{K}\right) \simeq 1.2$, a statement that they find depends very little on the gauge coupling $\beta$, at least in a range that covers our two simulations. This result is obtained with the lattice spacing fixed by the mass of the $\rho$ meson. With the lattice spacing set in this way, it is straightforward to show that $a \tilde{m}_{s}\left(m_{K^{*}}\right) \simeq a \tilde{m}_{s}\left(m_{\phi}\right)$. This follows from the observed linear behavior of the light vector meson masses and the fact that $m_{\rho} \simeq 2 m_{K^{*}}-m_{\phi}$ in nature. The picture changes when the value of the lattice spacing differs from the one given by the mass of the $\rho, a\left(m_{\rho}\right)$. In the present case, however, the values of the lattice spacing that we use appear to be consistent, within errors, with the values of $a\left(m_{\rho}\right)$ that can be inferred from Ref. [75]. Thus, we consider that the $20 \%$ upward variation in $a \tilde{m}_{s}$ described in Ref. [75] is a reasonable estimate of the uncertainty associated with the different possible choices of an observable to fix this mass. The changes that this variation induces in our results are reflected in the seventh error on the quantities in Tables VII, VIII and IX.

\section{F. Quenching errors}

Quenching effects for the quantities of interest here have been studied using quenched chiral perturbation theory [69]. They are typically a few percent for the $B$ parameters if the theory's couplings are constrained by large- $N_{c}$ arguments and by the reasonable range of Ref. 69, and larger outside these ranges. Recent results for the decay constants, obtained with two flavors of dynamical quarks $\left(n_{f}=2\right)$, show little variation in $f_{B_{s}} / f_{B}$ compared to its quenched value [38 41]. The authors of [38] find that this ratio is enhanced by $(5 \pm 3) \%$ in their calculation with light dynamical quarks. Thus, if quenching effects on the $B$ parameters are small, commensurate variations on $r_{s d}$ and $\xi$ are expected. For the decay constants of $B\left(B_{s}\right)$ mesons, quenching effects appear to be significant 38 41. For instance, the authors of Ref. [38] find that these decay constants are enhanced by $11 \%$ (14\%) when light-quark loops are included, with a

\footnotetext{
${ }^{17}$ Some of the baryons considered in Ref. 74. would give lattice spacings outside our $\pm 7 \%$. However, these particles are more susceptible, than the particles we are studying here, to systematic effects such as those associated with the finite volume of the lattice.
} 
statistical significance of 2 to 3 standard deviations. For the $D\left(D_{s}\right)$ mesons, the effect is of $3 \%(7 \%)$ and consistent with zero within roughly one standard deviation. These latter results suggest that quenching errors, at least on the $B$-meson decays constants, may be reduced by normalizing these constants with $f_{D_{s}}$. The reduction of quenching effects is about $7 \%$ and the remaining effects become consistent with zero. To the extent that quenching errors on the $B$ parameters are negligible, all of these considerations carry over to the quantities in Table IX which are proportional to decay constants. It should be remembered, however, that the real world has a third dynamical light quark and the effects discussed above may be amplified.

A thorough estimate of quenching effects for all of the quantities that we calculate would require a dedicated unquenched simulation, which is beyond the scope of this paper. Therefore, we do not attempt to quantify these effects specifically. Nevertheless, as we mentioned in the two previous sections, the uncertainties in the lattice scale and in the strange quark mass are, at least in part, quenching effects.

\section{FINAL RESULTS AND DISCUSSION}

Because of the excellent consistency of the results for $B_{B_{s}}, B_{B_{d}}, B_{B_{s}} / B_{B_{d}}$ and $f_{B_{s}} / f_{B_{d}}$ at the two lattice spacings and because we cannot perform a continuum extrapolation with only two points, we take the results from the finer lattice at $\beta=6.2$, which should have smaller discretization and matching uncertainties, to be our best estimates.

For the $S U(3)$-breaking ratio $r_{s d}$, we also have a choice between the "direct" and "indirect" approaches described in the Introduction. While both methods give results that are compatible at the two values of the lattice spacing, the "direct" method leads to larger variations with lattice spacing and significantly larger statistical errors. Furthermore, as discussed in Sections $\mathrm{V}$ and V1, the light- and heavy-quark-mass extrapolations are better behaved in the "indirect" method. Therefore, we take $r_{s d}$ obtained with the "indirect" method at $\beta=6.2$ as our best estimate for this quantity.

A summary of our results for quantities directly relevant for $B-\bar{B}$ mixing is given in Eq. (8). These results are compatible with previous calculations of some or all of these quantities, which were performed using less improved relativistic fermion actions [13, 23], as well as with the recent calculation of Ref. [24], which makes use of a nonperturbatively, $\mathcal{O}(a)$-improved, Sheikholeslami-Wohlert action. While the decay constants in Ref. [24] are nonperturbatively improved, 18 parameters and four-quark matrix elements are not. Thus, for those quantities, the discretization accuracy of that calculation is formally the same as ours. Moreover, the authors of Ref. 24 do not investigate cutoff dependence, as we do here with our two lattice spacings. Our results are also consistent, once systematic errors are taken into account, with the NRQCD results of Refs. 25, 26, whose $B$ parameters are $7 \sim 10 \%$ smaller than ours.

For the decay constants and quantities proportional to them, the situation is less favorable than for $B$ parameters and $S U$ (3)-breaking ratios. We do observe a two-statistical-standard-deviation dependence on lattice spacing, indicating that discretization errors are more important here. We quantify these discretization effects, as described in Section VII A. The corresponding uncertainty at $\beta=6.0$ is large enough to bridge the gap between the results for $B$-meson decay constants at the two lattice spacings. For $D$-meson decay constants, agreement requires that one also take into account the statistical error on the $\beta=6.2$ results. So we take as our best estimates the results from the finer lattice, which in principle have smaller discretization and matching uncertainties, confident that our errors are a reasonable estimate of the uncertainty associated with this fixed lattice spacing calculation. In the future, though, when $f_{D_{s}}$ is accurately measured experimentally, it will be advantageous to consider the values of these quantities in units of this decay constant. With this normalization, the discrepencies between the results at $\beta=6.2$ and 6.0 , as well as the size of systematic (and statistical) errors, are significantly reduced, as can be seen in Tables VII and IX.

Our results for the decay constants are summarized in Eq. (9). They are compatible with other recent calculations in the quenched approximation [32 36, 38, 39, as reviewed in Refs. 44, 46, 47]. A very recent, non-perturbatively $\mathcal{O}(a)$ improved calculation by the UKQCD Collaboration [37 yields $f_{B_{s}}$ and $f_{B}$ which are over two statistical standard deviations higher than our results. The authors of Ref. [37] use the scale $r_{0}$ [76] to set the inverse lattice spacing, $a^{-1}$, which gives an $a^{-1}$ at the top of our range. They further use the preliminary non-perturbative values of $b_{A}$ obtained in [77,78] and the non-perturbative values of $c_{A}$ obtained in [79]. [9] Agreement with our results is recovered,

\footnotetext{
${ }^{18}$ Almost: the authors actually use the perturbative value of $b_{A}$.

${ }^{19}$ The authors of Ref. [77,78] have since finalized their determinations of $b_{A}$, as well as those of $c_{A}$ [80]. The authors of Ref. 37] are currently reviewing their analysis of decay constants to incorporate, amongst other considerations, this new information 831 .
} 
nonetheless, when systematic errors are considered. For $D$-meson decay constants, their results agree with ours within statistical errors.

All of our results are obtained in the quenched approximation. Some of the error resulting from this approximation is accounted for by the variations that the uncertainties in the lattice spacing and the strange quark mass induce, since these uncertainties are, at least in part, quenching effects. However, a thorough estimate of quenching effects for all the quantities that we consider here would require a dedicated unquenched simulation, which is beyond the scope of this paper.

\section{CONCLUSION}

We have reported on high statistics, quenched lattice calculations of matrix elements relevant for neutral meson mixing, and leptonic decays of $B$ and $D$ mesons. We use mean-field-improved Sheikholeslami-Wohlert actions to describe quarks and work at two values of the lattice spacing. We have performed an extensive study of systematic errors and we believe that our final results, presented in Eqs. (8), (9) and (10), carry errors that reflect conservatively the uncertainty associated with our fixed lattice spacing calculations.

Our results for neutral $B$-meson mixing are compatible with the results of other calculations of some or all of the quantities we consider [13, 23, 26], as well as with the world averages of [17,42 47]. The same is true of our results for the decay constants, which are compatible with other modern, quenched determinations [32 36, 38, 39] and the world averages of [17, 42, 47].

Finally, it should be emphasized that all of these results are obtained in the quenched approximation. They include a quenching error only to the extent that the variations in the lattice spacing and strange quark mass that we account for are quenching effects. It is worth noting that when dimensionless quantities are considered, such as $B$ parameters, $S U(3)$-breaking ratios or quantities normalized by $f_{D_{s}}$, a number of systemaitc uncertainties, including possibly those associated with quenching, partially cancel. Nevertheless, a thorough quantification of quenching effects for neutral $B$-meson mixing matrix elements would require a dedicated unquenched simulation, which is beyond the scope of this paper. It is important, however, that such a study be undertaken. The pioneering $n_{f}=2$ studies of decay constants [38 41] are a first step in this direction.

\section{Acknowledgments}

This work is supported by EPSRC and PPARC under Grants Nos. GR/K41663 and GR/L29927. We acknowledge the help from the UKQCD Collaboration, especially Pablo Martinez and Peter Boyle, on generating the lattice data. We thank Damir Becirevic, Ken Bowler, Jonathan Flynn, Leonardo Giusti, Richard Kenway, Vittorio Lubicz, Craig McNeile, Guido Martinelli, Chris Maynard, Brian Pendleton, David Richards, Chris Sachrajda and Hartmut Wittig for useful discussions. L. L. thanks the University of Edinburgh and C.-J. D. L. thanks Centre de Physique Théorique of CNRS Luminy and the Theory Division of CERN for their hospitality. C.-J. D. L. acknowledges the financial support of the Department of Physics and Astronomy of the University of Kentucky under DOE EPSCor grant DE-FG05-84ER40154.

[1] A. Stocchi (2000), hep-ph/0010222.

[2] M. Daoudi, in the proceedings of the 8th International Symposium on Heavy Flavor Physics (Heavy Flavors 8), Southampton, England, 25-29 July 1999, hep-ex/9911027.

[3] A. Buras, M. Jamin, and P. Weisz, Nucl. Phys. B347, 491 (1990).

[4] G. Buchalla, A. J. Buras, and M. E. Lautenbacher, Rev. Mod. Phys. 68, 1125 (1996), hep-ph/9512380.

[5] T. Inami and C. Lim, Prog. Theor. Phys 65, 297 (1981), erratum ibid. 65, 1772 (1981).

[6] For a recent review see, for instance, R. A. Poling, in the proceedings of the 19th International Symposium on Lepton and Photon Interactions at High-Energies (LP 99), Stanford, California, 9-14 August 1999, hep-ex/0003025.

[7] A. J. Buras, in Santa Barbara 1997, Heavy Flavor Physics, pp. 76-106, hep-ph/9711217.

[8] S. Mele, Phys. Rev. D59, 113011 (1999), hep-ph/9810333.

[9] A. Ali and D. London, Eur. Phys. J. C9, 687 (1999), hep-ph/9903535. 
[10] M. Ciuchini, E. Franco, L. Giusti, V. Lubicz, and G. Martinelli, Nucl. Phys. B573, 201 (2000), hep-ph/9910236.

[11] S. Plaszczynski and M. Schune, talk given at the 8th International Symposium on Heavy Flavor Physics (Heavy Flavors 8), Southampton, England, 25-29 July 1999, hep-ph/991128.

[12] F. Caravaglios, F. Parodi, P. Roudeau, and A. Stocchi, talk given at the 3rd International Conference on B Physics and CP Violation (BCONF99), Taipei, Taiwan, 3-7 December 1999, hep-ph/0002171.

[13] C. Bernard, T. Blum, and A. Soni, Phys. Rev. D58, 014501 (1998), hep-lat/9801039.

[14] G. P. Lepage and P. B. Mackenzie, Phys. Rev. D48, 2250 (1993), hep-lat/9209022.

[15] B. Sheikholeslami and R. Wohlert, Nucl. Phys. B259, 572 (1985).

[16] L. Lellouch and C. J. D. Lin (UKQCD), Nucl. Phys. Proc. Suppl. 73, 357 (1999), hep-lat/9809018.

17] L. Lellouch, invited talk given at the 34th Rencontres de Moriond: Electroweak Interactions and Unified Theories, Les Arcs, France, 13-20 March 1999, hep-ph/9906497.

[18] UKQCD Collaboration, L. Lellouch and C.-J.D. Lin, contribution to the 8th International Symposium on Heavy Flavor Physics (Heavy Flavors 8), Southampton, England, 25-29 July 1999, hep-ph/9912322.

[19] F. Parodi, P. Roudeau, and A. Stocchi, Nuovo Cim. A112, 833 (1999), hep-ex/9903063.

[20] ALEPH Collaboration, contributed to the 30th International Conference on High-Energy Physics (ICHEP 2000), Osaka, Japan, 27 July - 2 August, ALEPH 2000-062.

[21] Y. Alexandrov et al. (BEATRICE), Phys. Lett. B478, 31 (2000).

[22] M. Chadha et al. (CLEO), Phys. Rev. D58, 032002 (1998), hep-ex/9712014.

[23] S. Aoki et al. (JLQCD), Nucl. Phys. Proc. Suppl. 47, 433 (1996), hep-lat/9510033.

[24] D. Becirevic et al. (2000), hep-lat/0002025.

[25] S. Hashimoto et al., Phys. Rev. D62, 114502 (2000), hep-lat/0004022.

[26] N. Yamada et al. (JLQCD), Nucl. Phys. Proc. Suppl. 94, 379 (2001), hep-lat/0010089.

[27] A. K. Ewing et al. (UKQCD), Phys. Rev. D54, 3526 (1996), hep-lat/9508030.

[28] V. Gimenez and G. Martinelli, Phys. Lett. B398, 135 (1997), hep-lat/9610024.

[29] J. Christensen, T. Draper, and C. McNeile, Phys. Rev. D56, 6993 (1997), hep-lat/9610026.

[30] V. Gimenez and J. Reyes, Nucl. Phys. B545, 576 (1999), hep-lat/9806023.

[31] D. Becirevic et al., in preparation.

[32] C. R. Allton et al., Phys. Lett. B405, 133 (1997), hep-lat/9703002.

[33] A. X. El-Khadra, A. S. Kronfeld, P. B. Mackenzie, S. M. Ryan, and J. N. Simone, Phys. Rev. D58, 014506 (1998), hep-ph/9711426.

[34] A. Ali Khan et al., Phys. Lett. B427, 132 (1998), hep-lat/9801038.

[35] C. Bernard et al., Phys. Rev. Lett. 81, 4812 (1998), hep-ph/9806412.

[36] K.-I. Ishikawa et al. (JLQCD), Phys. Rev. D61, 074501 (2000), hep-lat/9905036.

[37] K. C. Bowler et al. (UKQCD) (2000), hep-lat/0007020.

[38] A. Ali Khan et al. (CP-PACS) (2000), hep-lat/0010009.

[39] C. Bernard et al., Nucl. Phys. Proc. Suppl. 94, 346 (2001), hep-lat/0011029.

[40] C. Bernard et al. (MILC), Nucl. Phys. Proc. Suppl. 83, 289 (2000), hep-lat/9909121.

[41] S. Collins et al., Phys. Rev. D60, 074504 (1999), hep-lat/9901001.

[42] H. Wittig, lectures given at the International School of Physics, "Enrico Fermi": Heavy Flavor Physics - A Probe of Nature's Grand Design, Varenna, Italy, 8-18 July 1997, hep-lat/9710088.

[43] J. M. Flynn and C. T. Sachrajda, in Buras, A.J. (ed.), Lindner, M. (ed.): Heavy flavours II, World Scientific, Singapore (1992) pp. 402-452, hep-lat/9710057.

[44] T. Draper, Nucl. Phys. Proc. Suppl. 73, 43 (1999), hep-lat/9810065.

[45] S. Sharpe, talk given at the 29th International Conference on High-Energy Physics (ICHEP 98), Vancouver, Canada, 23-29 July 1998, hep-lat/9811006.

[46] S. Hashimoto, Nucl. Phys. Proc. Suppl. 83, 3 (2000), hep-lat/9909136.

[47] V. Lubicz (2000), hep-ph/0010171.

[48] A. Datta and D. Kumbhakar, Z. Phys. C27, 515 (1985).

[49] R. Barbieri and R. Gatto, Phys. Lett. B110, 211 (1982).

[50] J. Ellis and D. V. Nanopoulos, Phys. Lett. B110, 44 (1982).

[51] F. Gabbiani and A. Masiero, Nucl. Phys. B322, 235 (1989).

[52] Y. Nir and N. Seiberg, Phys. Lett. B309, 337 (1993), hep-ph/9304307.

[53] M. Leurer, Y. Nir, and N. Seiberg, Nucl. Phys. B420, 468 (1994), hep-ph/9310320.

[54] J. F. Donoghue, E. Golowich, B. R. Holstein, and J. Trampetic, Phys. Rev. D33, 179 (1986).

[55] C. R. Allton et al. (UKQCD), Nucl. Phys. B407, 331 (1993), hep-lat/9307009.

[56] G. Heatlie, G. Martinelli, C. Pittori, G. C. Rossi, and C. T. Sachrajda, Nucl. Phys. B352, 266 (1991).

[57] H. P. Shanahan et al. (UKQCD), Phys. Rev. D55, 1548 (1997), hep-lat/9608063.

[58] M. Lüscher, S. Sint, R. Sommer, P. Weisz, and U. Wolff, Nucl. Phys. B491, 323 (1997), hep-lat/9609035.

[59] A. X. El-Khadra, A. S. Kronfeld, and P. B. Mackenzie, Phys. Rev. D55, 3933 (1997), hep-lat/9604004.

[60] P. Lacock, A. McKerrell, C. Michael, I. M. Stopher, and P. W. Stephenson (UKQCD), Phys. Rev. D51, 6403 (1995), 
hep-lat/9412079.

[61] B. Efron, The Jacknife, the Bootstrap and other Resampling Plans (Soc. for Industrial and Applied mathematics, Philadelphia, 1982).

[62] E. Gabrielli, G. Martinelli, C. Pittori, G. Heatlie, and C. T. Sachrajda, Nucl. Phys. B362, 475 (1991).

[63] S. Capitani, M. Gockeler, R. Horsley, H. Perlt, P. Rakow, G. Schierholz, and A. Schiller, Nucl. Phys. (Proc. Suppl.) 63, 874 (1998).

[64] R. Frezzotti, E. Gabrielli, C. Pittori, and G. C. Rossi, Nucl. Phys. B373, 781 (1992).

[65] R. Gupta, T. Bhattacharya, and S. Sharpe, Phys. Rev. D55, 4036 (1997), hep-lat/9611023.

[66] M. Lüscher, S. Sint, R. Sommer, and P. Weisz, Nucl. Phys. B478, 365 (1996), hep-lat/9605038.

[67] S. R. Sharpe, Phys. Rev. D46, 3146 (1992), hep-lat/9205020.

[68] C. W. Bernard and M. F. L. Golterman, Phys. Rev. D46, 853 (1992), hep-lat/9204007.

[69] S. R. Sharpe and Y. Zhang, Phys. Rev. D53, 5125 (1996), hep-lat/9510037.

[70] M. Voloshin and M. Shifman, Sov. J. Phys. 45, 292 (1987).

[71] H. Politzer and M. Wise, Phys. Lett. B 206, 681 (1988); ibid. 208, 504 (1988).

[72] M. Lüscher and P. Weisz, Nucl. Phys. B479, 429 (1996), hep-lat/9606016.

[73] S. Sint and P. Weisz, Nucl. Phys. B502, 251 (1997), hep-lat/9704001.

[74] K. C. Bowler et al. (UKQCD), Phys. Rev. D62, 054506 (2000), hep-lat/9910022.

[75] T. Bhattacharya and R. Gupta, Nucl. Phys. Proc. Suppl. 63, 95 (1998), hep-lat/9710095.

[76] R. Sommer, Nucl. Phys. B411, 839 (1994), hep-lat/9310022.

[77] T. Bhattacharya, S. Chandrasekharan, R. Gupta, W. Lee, and S. Sharpe, Phys. Lett. B461, 79 (1999), hep-lat/9904011.

[78] T. Bhattacharya, R. Gupta, W. Lee, and S. Sharpe, Nucl. Phys. Proc. Suppl. 83, 851 (2000), hep-lat/9909115.

[79] M. Lüscher et al., Nucl. Phys. Proc. Suppl. 53, 905 (1997), hep-lat/9608049.

[80] T. Bhattacharya, R. Gupta, W. Lee, and S. Sharpe, Phys. Rev. D D63, 074505 (2001), hep-lat/0009038.

[81] C. Maynard, private communication.

TABLE I. Simulation parameters. $c_{\mathrm{SW}}$ is the clover coefficient.

\begin{tabular}{ccc}
\hline \hline$\beta$ & 6.2 & 6.0 \\
\hline$c_{\mathrm{SW}}$ & 1.442 & 1.479 \\
size & $24^{3} \times 48$ & $16^{3} \times 48$ \\
\# of configurations & 188 & 498 \\
\hline \hline
\end{tabular}

TABLE II. Hopping parameters, $\kappa_{\psi}$, of the light $(\psi=q)$ and heavy $(\psi=Q)$ quarks used in the simulations. In brackets, we give the masses, $m_{p}$, of light-light pseudoscalar mesons composed of a degenerate quark and antiquark with hopping parameter $\kappa_{q}$, obtained as described in Section IV. We also provide the masses, $M_{P}$, of heavy-light pseudoscalar mesons composed of a heavy quark with hopping parameter $\kappa_{Q}$ and a massless antiquark, obtained as described in Section IV] and Section VI. Error bars are statistical only. The scales used to translate these masses into $\mathrm{MeV}$ are $a^{-1}=2.73 \mathrm{GeV}$ at $\beta=6.2$ and $a^{-1}=2.00 \mathrm{GeV}$ at $\beta=6.0$, as obtained in Section $\mathrm{V}$.

\begin{tabular}{ccc}
\hline \hline$\beta$ & $\kappa_{q}\left[m_{p}\right.$ in $\left.\mathrm{MeV}\right]$ & $\kappa_{Q}\left[M_{P}\right.$ in $\left.\mathrm{MeV}\right]$ \\
\hline 6.2 & $0.13640[831(4)], 0.13710[608(4)]$, & $0.120[2238(14)], 0.123[2006(13)]$, \\
& $0.13745[466(6)]$ & $0.126[1757(11)], 0.129[1488(10)]$ \\
& $0.132[1186(9)]$ \\
\hline 6.0 & $0.13700[827(1)], 0.13810[587(3)]$, & $0.114[2183(6)], 0.118[1971(5)]$, \\
& $0.13856[454(3)]$ & $0.122[1746(4)], 0.126[1503(4)]$, \\
& $0.130[1234(3)]$ \\
\hline \hline
\end{tabular}


TABLE III. The coupling $\alpha_{\overline{\mathrm{MS}}}(q)$ at different values of $q$ as obtained in the simulations at $\beta=6.0$ and 6.2 .

\begin{tabular}{cccc}
\hline \hline$\beta$ & $\alpha_{\overline{\mathrm{MS}}}(1 / a)$ & $\alpha_{\overline{\mathrm{MS}}}(2 / a)$ & $\alpha_{\overline{\mathrm{MS}}}(\pi / a)$ \\
\hline 6.2 & 0.1730 & 0.1402 & 0.1250 \\
6.0 & 0.1921 & 0.1522 & 0.1343 \\
\hline \hline
\end{tabular}

TABLE IV. Fit ranges for the correlation functions and ratios used in this work.

\begin{tabular}{ccc}
\hline \hline$\beta$ & 6.2 & 6.0 \\
\hline$C_{\mathcal{O}}^{F L F} / C_{A P}^{L F} C_{A P}^{L F}$ & $34 \leq t_{x} \leq 38$ & $33 \leq t_{x} \leq 37$ \\
& $10 \leq t_{y} \leq 14$ & $11 \leq t_{y} \leq 15$ \\
\hline$C_{\mathcal{O}}^{F L F} / C_{P P}^{F F} C_{P P}^{F F}$ & $34 \leq t_{x} \leq 38$ & $33 \leq t_{x} \leq 37$ \\
& $10 \leq t_{y} \leq 14$ & $11 \leq t_{y} \leq 15$ \\
\hline$C_{P A}^{F L}\left(t_{x}\right) / C_{P P}^{F F}\left(t_{x}\right)$ & $15 \leq t_{x} \leq 23$ & $13 \leq t_{x} \leq 23$ \\
\hline$C_{P P}^{F F}$ & $13 \leq t_{x} \leq 23$ & $11 \leq t_{x} \leq 23$ \\
\hline$C_{P P}^{F L}$ & $13 \leq t_{x} \leq 23$ & $11 \leq t_{x} \leq 23$ \\
\hline$C_{p p}^{F L}$ & $10 \leq t_{x} \leq 20$ & $5 \leq t_{x} \leq 23$ \\
\hline$C_{p a}^{F L}\left(t_{x}\right) / C_{p p}^{F F}\left(t_{x}\right)$ & $15 \leq t_{x} \leq 2311 \leq t_{x} \leq 23$ \\
\hline \hline
\end{tabular}

TABLE V. Lattice spacings, critical hopping parameters and bare, strange quark masses, obtained as detailed in the text. Errors are statistical.

\begin{tabular}{ccc}
\hline \hline$\beta$ & 6.2 & 6.0 \\
\hline$a^{-1}(\mathrm{GeV})$ & $2.73_{-8}^{+10}$ & $2.00_{-4}^{+4}$ \\
$\kappa_{c r}$ & $0.13792(2)$ & $0.13921(1)$ \\
$a \tilde{m}_{s}$ & $0.0280(19)$ & $0.0401(17)$ \\
\hline \hline
\end{tabular}


TABLE VI. Results for the fit parameters for the heavy-quark-mass dependence of the various quantities studied. $C_{X}=0$ indicates a linear fit and $C_{X}=B_{X}=0$, a fit to a constant.

\begin{tabular}{ccccc}
\hline \hline & $\beta$ & $A_{X}$ & $B_{X}$ & $C_{X}$ \\
\hline$\Phi_{B}^{s}\left(M_{P_{l}}\right)$ & 6.2 & $0.94(2)$ & $-0.08(1)$ & 0 \\
& 6.0 & $0.95(2)$ & $-0.10(2)$ & 0 \\
\hline$\Phi_{B}^{l}\left(M_{P_{l}}\right)$ & 6.2 & $0.96(4)$ & $-0.10(2)$ & 0 \\
& 6.0 & $0.93(5)$ & $-0.12(3)$ & 0 \\
\hline$B_{P_{s}} / B_{P_{d}}$ & 6.2 & $0.97(3)$ & $0.02(1)$ & 0 \\
& 6.0 & $1.01(4)$ & $0.03(2)$ & 0 \\
\hline$\Phi_{\Delta F=2}^{s}\left(M_{P_{l}}\right)$ & 6.2 & $0.010(1)$ & $-0.29(1)$ & 0 \\
& 6.0 & $0.058(4)$ & $-0.85(2)$ & $0.22(1)$ \\
\hline$\Phi_{\Delta F=2}^{l}\left(M_{P_{l}}\right)$ & 6.2 & $0.006(1)$ & $-0.29(2)$ & 0 \\
& 6.0 & $0.041(6)$ & $-0.83(6)$ & $0.20(3)$ \\
\hline$\left(\left\langle P_{s}\left|\mathcal{O}_{s}^{\Delta F=2}\right| P_{s}\right\rangle /\left\langle P_{d}\left|\mathcal{O}_{d}^{\Delta F=2}\right| P_{d}\right\rangle\right)$ & 6.2 & $1.57(26)$ & $0.01(6)$ & 0 \\
$\times\left(M_{P_{d}} / M_{P_{s}}\right)$ & 6.0 & $1.28(14)$ & $0.11(8)$ & 0 \\
\hline$\Phi_{f}^{s}\left(M_{P_{l}}\right)$ & 6.2 & $0.10(1)$ & $-0.32(4)$ & $0.04(1)$ \\
& 6.0 & $0.19(1)$ & $-0.53(2)$ & $0.11(1)$ \\
\hline$\Phi_{f}^{l}\left(M_{P_{l}}\right)$ & 6.2 & $0.09(2)$ & $-0.30(8)$ & $0.04(2)$ \\
& 6.0 & $0.17(1)$ & $-0.51(4)$ & $0.11(2)$ \\
\hline$\left(f_{P_{s}} / f_{P_{l}}\right)$ & 6.2 & $1.16(2)$ & 0 & 0 \\
$\times \sqrt{M_{P_{s}} / M_{P_{l}}}$ & 6.0 & $1.15(1)$ & 0 & 0 \\
\hline \hline
\end{tabular}

TABLE VII. Results at the two values of the lattice spacing. The first error on each quantity is statistical while the others correspond, respectively, to the variations in procedure described in the first five subsections in Section VII. The errors enclosed in brackets reflect the variations considered in quantifying discretization errors.

\begin{tabular}{|c|c|c|c|c|c|c|c|c|c|}
\hline $\bar{\beta}$ & \multicolumn{4}{|c|}{6.2} & \multicolumn{5}{|c|}{6.0} \\
\hline$f_{B}[\mathrm{MeV}]$ & $177_{-16}^{+17}$ & $\begin{array}{ll}+8 & +11 \\
-5 & -0\end{array}$ & $\begin{array}{lll}+6 & +0 & +16 \\
-3 & -6 & -16\end{array}$ & $\begin{array}{l}+0 \\
-0\end{array}$ & $205_{-8}^{+9}$ & $\begin{array}{ll}+14 & +23 \\
-9 & -0\end{array}$ & $\begin{array}{l}+9 \\
-4\end{array}$ & $\begin{array}{ll}+0 & +18 \\
-11 & -18\end{array}$ & $\begin{array}{ll}8 & +0 \\
8 & -0\end{array}$ \\
\hline$\frac{f_{B}}{f_{D_{s}}}$ & $0.71_{-6}^{+6}$ & $\begin{array}{ll}+2 & +3 \\
-1 & -0\end{array}$ & $\begin{array}{lll}+0 & +0 & +3 \\
-0 & -2 & -3\end{array}$ & $\begin{array}{l}+0 \\
-2\end{array}$ & $0.76_{-3}^{+3}$ & $\begin{array}{ll}+2 & +6 \\
-1 & -0\end{array}$ & $\begin{array}{l}+0 \\
-0\end{array}$ & $\begin{array}{lll}+0 & +3 \\
-4 & -3\end{array}-$ & $\begin{array}{l}+0 \\
-2\end{array}$ \\
\hline$f_{B_{s}}[\mathrm{MeV}]$ & $204 \begin{array}{l}+12 \\
-12\end{array}$ & $\begin{array}{ll}+10 & +13 \\
-6 & -0\end{array}$ & $\begin{array}{lll}+8 & +0 & +14 \\
-3 & -7 & -1\end{array}$ & $\begin{array}{ll}4 & +6 \\
4 & -0\end{array}$ & $233_{-5}^{+5}$ & $\begin{array}{ll}+17 & +27 \\
-11 & -0\end{array}$ & $\begin{array}{l}+10 \\
-4\end{array}$ & $\begin{array}{ll}+0 & +16 \\
-13 & -16\end{array}$ & $\begin{array}{ll}16 & +6 \\
16 & -0\end{array}$ \\
\hline$\frac{f_{B_{s}}}{f_{D_{s}}}$ & $0.82_{-3}^{+3}$ & $\left.\begin{array}{ll}+2 & +4 \\
-1 & -0\end{array}\right]$ & $\begin{array}{lll}+0 & +0 & +2 \\
-0 & -3 & -2\end{array}$ & $\begin{array}{l}+0 \\
-0\end{array}$ & $0.87_{-1}^{+1}$ & $\begin{array}{ll}+3 & +7 \\
-1 & -0\end{array}$ & $\begin{array}{l}+0 \\
-0\end{array}$ & $\begin{array}{ll}+0 & +2 \\
-5 & -2\end{array}$ & $\begin{array}{l}+0 \\
-0\end{array}$ \\
\hline$\frac{f_{B_{s}}}{f_{B}}$ & $1.15_{-2}^{+2}$ & $\begin{array}{ll}+0 & +0 \\
-0 & -0\end{array}$ & $\begin{array}{lll}+0 & +0 & +3 \\
-0 & -0 & -2\end{array}$ & $\begin{array}{l}+3 \\
-0\end{array}$ & $1.14_{-1}^{+1}$ & $\begin{array}{ll}+0 & +0 \\
-0 & -0\end{array}$ & $\begin{array}{l}+0 \\
-0\end{array}$ & $\begin{array}{ll}+0 & +2 \\
-0 & -2\end{array}$ & $\begin{array}{l}+3 \\
-0\end{array}$ \\
\hline$f_{D}[\mathrm{MeV}]$ & $210_{-9}^{+10}$ & $\left.\begin{array}{ll}+5 & +4 \\
-4 & -0\end{array}\right]$ & $\begin{array}{lll}+8 & +0 & +14 \\
-3 & -0 & -15\end{array}$ & $\begin{array}{l}+0 \\
-0\end{array}$ & $228 \begin{array}{l}+4 \\
-4\end{array}$ & $\begin{array}{ll}+7 & +8 \\
-7 & -0\end{array}$ & $\begin{array}{l}+10 \\
-4\end{array}$ & $\begin{array}{ll}+0 & +14 \\
-0 & -15\end{array}$ & $\begin{array}{l}+0 \\
-0\end{array}$ \\
\hline$f_{D_{s}}[\mathrm{MeV}]$ & $236_{-8}^{+8}$ & $\begin{array}{ll}+6 & +4 \\
-5 & -0\end{array}$ & $\begin{array}{lll}+9 & +0 & +12 \\
-3 & -0 & -12\end{array}$ & $\begin{array}{l}+7 \\
-0\end{array}$ & $254 \begin{array}{l}+3 \\
-3\end{array}$ & $\begin{array}{ll}+9 & +9 \\
-8 & -0\end{array}$ & $\begin{array}{l}+11 \\
-5\end{array}$ & $\begin{array}{ll}+0 & +11 \\
-0 & -12\end{array}$ & $\begin{array}{l}+7 \\
-0\end{array}$ \\
\hline$\frac{f_{D_{s}}}{f_{D}}$ & $1.13_{-2}^{+2}$ & $\begin{array}{ll}+0 & +0 \\
-0 & -0\end{array}$ & $\begin{array}{lll}+0 & +0 & +3 \\
-0 & -0 & -2\end{array}$ & $\begin{array}{l}+3 \\
-0\end{array}$ & $1.11_{-1}^{+1}$ & $\begin{array}{ll}+0 & +0 \\
-0 & -0\end{array}$ & $\begin{array}{l}+0 \\
-0\end{array}$ & $\begin{array}{ll}+0 & +2 \\
-0 & -2\end{array}$ & $\begin{array}{l}+3 \\
-0\end{array}$ \\
\hline
\end{tabular}


TABLE VIII. Results at the two values of the lattice spacing (continued). $r_{s d}^{\text {direct }}=\left\langle\bar{B}_{s}\left|\mathcal{O}_{s}^{\Delta B=2}\right| B_{s}\right\rangle /\left\langle\bar{B}_{d}\left|\mathcal{O}_{d}^{\Delta B}=2\right| B_{d}\right\rangle$. An error of $x$ indicates that the variation has not been explicitly performed, but that it is believed to be small. An error of $y$ means that the variation has not been explicitely performed.

\begin{tabular}{|c|c|c|c|c|c|c|c|c|c|c|c|c|c|c|}
\hline$\beta$ & \multicolumn{7}{|c|}{6.2} & \multicolumn{7}{|c|}{6.0} \\
\hline$B_{B_{d}}\left(M_{B}\right)$ & $0.91_{-4}^{+3}$ & $\begin{array}{l}+x \\
-x\end{array}$ & $\begin{array}{l}+0 \\
-0\end{array}$ & $\begin{array}{l}+4 \\
-0\end{array}$ & $\begin{array}{l}+x \\
-x\end{array}$ & $\begin{array}{l}+0 \\
-0\end{array}$ & $\begin{array}{l}+0 \\
-0\end{array}$ & $0.89_{-4}^{+4}$ & $\begin{array}{l}+x \\
-x\end{array}$ & $\begin{array}{l}+0 \\
-0\end{array}$ & $\begin{array}{l}+3 \\
-0\end{array}$ & $\begin{array}{l}+0 \\
-0\end{array}$ & $\begin{array}{l}+0 \\
-0\end{array}$ & $\begin{array}{l}+0 \\
-0\end{array}$ \\
\hline$B_{B_{s}}\left(M_{B}\right)$ & $0.90_{-2}^{+2}$ & $\begin{array}{l}+x \\
-x\end{array}$ & $\begin{array}{l}+0 \\
-0\end{array}$ & $\begin{array}{l}+3 \\
-0\end{array}$ & $\begin{array}{l}+x \\
-x\end{array}$ & $\begin{array}{l}+0 \\
-0\end{array}$ & $\begin{array}{l}+0 \\
-0\end{array}$ & $0.91_{-2}^{+2}$ & $\begin{array}{l}+x \\
-x\end{array}$ & $\begin{array}{l}+0 \\
-0\end{array}$ & $\begin{array}{l}+3 \\
-0\end{array}$ & $\begin{array}{l}+0 \\
-0\end{array}$ & $\begin{array}{l}+1 \\
-1\end{array}$ & $\begin{array}{l}+0 \\
-0\end{array}$ \\
\hline$B_{D}\left(M_{D}\right)$ & $0.82_{-3}^{+3}$ & $\begin{array}{l}+x \\
-x\end{array}$ & $\begin{array}{l}+0 \\
-0\end{array}$ & $\begin{array}{l}+3 \\
-0\end{array}$ & $\begin{array}{l}+x \\
-x\end{array}$ & $\begin{array}{l}+1 \\
-1\end{array}$ & $\begin{array}{l}+0 \\
-0\end{array}$ & $0.81_{-3}^{+3}$ & $\begin{array}{l}+x \\
-x\end{array}$ & $\begin{array}{l}+0 \\
-0\end{array}$ & $\begin{array}{l}+2 \\
-0\end{array}$ & $\begin{array}{l}+0 \\
-0\end{array}$ & $\begin{array}{l}+1 \\
-1\end{array}$ & $\begin{array}{l}+0 \\
-0\end{array}$ \\
\hline$\frac{B_{B_{s}}}{B_{B_{d}}}$ & $0.98_{-2}^{+2}$ & $\begin{array}{l}+x \\
-x\end{array}$ & $\begin{array}{l}+0 \\
-0\end{array}$ & $\begin{array}{l}+0 \\
-2\end{array}$ & $\begin{array}{l}+x \\
-x\end{array}$ & $\begin{array}{l}+0 \\
-0\end{array}$ & $\begin{array}{l}+0 \\
-0\end{array}$ & $1.02 \begin{array}{l}+3 \\
-3\end{array}$ & $\begin{array}{l}+x \\
-x\end{array}$ & $\begin{array}{l}+0 \\
-0\end{array}$ & $\begin{array}{l}+0 \\
-0\end{array}$ & $\begin{array}{l}+0 \\
-0\end{array}$ & $\begin{array}{l}+0 \\
-0\end{array}$ & $\begin{array}{l}+0 \\
-0\end{array}$ \\
\hline$r_{s d}^{\text {direct }}$ & $1.61_{-20}^{+20}$ & $\begin{array}{l}+x \\
-x\end{array}$ & $\left.\begin{array}{l}+0 \\
-0\end{array}\right]$ & $\begin{array}{l}+10 \\
-0\end{array}$ & $\begin{array}{l}+y \\
-x\end{array}$ & $\begin{array}{l}+9 \\
-7\end{array}$ & $\begin{array}{l}+12 \\
-0\end{array}$ & $1.36_{-12}^{+11}$ & {$\left[\begin{array}{l}+x \\
-x\end{array}\right.$} & $\begin{array}{l}+0 \\
-0\end{array}$ & $\begin{array}{l}+1 \\
-0\end{array}$ & $\begin{array}{l}+6 \\
-0\end{array}$ & $\begin{array}{l}+8 \\
-0\end{array}$ & $\begin{array}{l}+7 \\
-0\end{array}$ \\
\hline
\end{tabular}

TABLE IX. Results at the two values of the lattice spacing (continued). $r_{s d}^{\text {indirect }}=\left(\frac{M_{B_{s}}}{M_{B_{d}}}\right)^{2}\left[\frac{f_{B_{s}}^{2} B_{B_{s}}}{f_{B_{d}}^{2} B_{B_{d}}}\right]$.

\begin{tabular}{|c|c|c|c|c|c|c|}
\hline $\bar{\beta}$ & \multicolumn{3}{|c|}{6.2} & \multicolumn{3}{|c|}{6.0} \\
\hline$f_{B_{d}} \sqrt{\hat{B}_{B_{d}}^{\text {nlo }}}[\mathrm{MeV}]$ & $210_{-21}^{+20}$ & $\begin{array}{ll}+10 & +13 \\
-6 & -0\end{array}$ & $\begin{array}{llll}+12 & +0 & +19 & +0 \\
-0 & -7 & -18 & -0\end{array}$ & $240_{-11}^{+12}$ & $\begin{array}{ll}+16 & +27 \\
-11 & -0\end{array}$ & $\begin{array}{llll}+15 & +0 & +21 & +0 \\
-5 & -13 & -21 & -0\end{array}$ \\
\hline$\frac{f_{B_{d}}}{f_{D_{s}}} \sqrt{\hat{B}_{B_{d}}^{\text {nlo }}}$ & $0.89_{-7}^{+7}$ & $\begin{array}{ll}+2 & +4 \\
-1 & -0\end{array}$ & $\begin{array}{llll}+2 & +0 & +3 & +0 \\
-0 & -3 & -4 & -2\end{array}$ & $0.95_{-4}^{+5}$ & $\begin{array}{ll}+3 & +7 \\
-1 & -0\end{array}$ & $\begin{array}{llll}+2 & +0 & +4 & +0 \\
-0 & -5 & -4 & -2\end{array}$ \\
\hline$f_{B_{s}} \sqrt{\hat{B}_{B_{s}}^{\text {nlo }}}[\mathrm{MeV}]$ & $241 \begin{array}{l}+14 \\
-14\end{array}$ & $\begin{array}{ll}+12 & +15 \\
-7 & -0\end{array}$ & $\begin{array}{llll}+13 & +0 & +17 & +7 \\
-1 & -9 & -16 & -0\end{array}$ & $277_{-6}^{+7}$ & $\begin{array}{ll}+20 & +32 \\
-13 & -0\end{array}$ & $\begin{array}{llll}+18 & +0 & +18 & +8 \\
-5 & -15 & -18 & -0\end{array}$ \\
\hline$\frac{f_{B_{s}}}{f_{D_{s}}} \sqrt{\hat{B}_{B_{s}}^{\text {nlo }}}$ & $1.02 \begin{array}{l}+4 \\
-4\end{array}$ & $\begin{array}{ll}+3 & +5 \\
-1 & -0\end{array}$ & $\begin{array}{llll}+2 & +0 & +2 & +0 \\
-0 & -4 & -2 & -0\end{array}$ & $1.09_{-2}^{+2}$ & $\begin{array}{ll}+4 & +9 \\
-2 & -0\end{array}$ & $\begin{array}{llll}+2 & +0 & +2 & +0 \\
-0 & -6 & -2 & -0\end{array}$ \\
\hline$f_{D} \sqrt{\hat{B}_{D}^{\text {nlo }}[\mathrm{MeV}]}$ & $222 \begin{array}{l}+10 \\
-10\end{array}$ & $\left.\begin{array}{ll}+5 & +5 \\
-4 & -0\end{array}\right]$ & $\begin{array}{llll}+13 & +0 & +14 & +0 \\
-0 & -0 & -14 & -0\end{array}$ & $240 \begin{array}{l}+7 \\
-6\end{array}$ & $\begin{array}{ll}+8 & +8 \\
-7 & -0\end{array}$ & $\begin{array}{llll}+13 & +0 & +14 & +0 \\
-3 & -0 & -14 & -0\end{array}$ \\
\hline$\frac{f_{D}}{f_{D_{s}}} \sqrt{\hat{B}_{D}^{\text {nlo }}}$ & $0.94_{-3}^{+3}$ & $\begin{array}{ll}+0 & +1 \\
-0 & -0\end{array}$ & $\begin{array}{llll}+2 & +0 & +1 & +0 \\
-0 & -0 & -1 & -3\end{array}$ & $0.94_{-2}^{+2}$ & $\begin{array}{ll}+0 & +0 \\
-0 & -0\end{array}$ & $\begin{array}{llll}+1 & +0 & +1 & +0 \\
-0 & -0 & -1 & -2\end{array}$ \\
\hline$\xi$ & $1.15_{-2}^{+2}$ & $\begin{array}{ll}+0 & +0 \\
-0 & -0\end{array}$ & $\begin{array}{llll}+0 & +0 & +2 & +3 \\
-1 & -0 & -2 & -0\end{array}$ & $1.16_{-2}^{+2}$ & $\begin{array}{ll}+0 & +0 \\
-0 & -0\end{array}$ & $\begin{array}{llll}+0 & +0 & +3 & +3 \\
-0 & -0 & -2 & -0\end{array}$ \\
\hline$r_{s d}^{\text {indirect }}$ & $1.38_{-5}^{+6}$ & $\begin{array}{ll}+1 & +0 \\
-0 & -0\end{array}$ & $\begin{array}{llll}+0 & +0 & +6 & +8 \\
-3 & -1 & -5 & -0\end{array}$ & $1.39_{-5}^{+5}$ & $\begin{array}{ll}+1 & +0 \\
-0 & -0\end{array}$ & $\begin{array}{llll}+0 & +0 & +6 & +8 \\
-0 & -0 & -5 & -0\end{array}$ \\
\hline
\end{tabular}




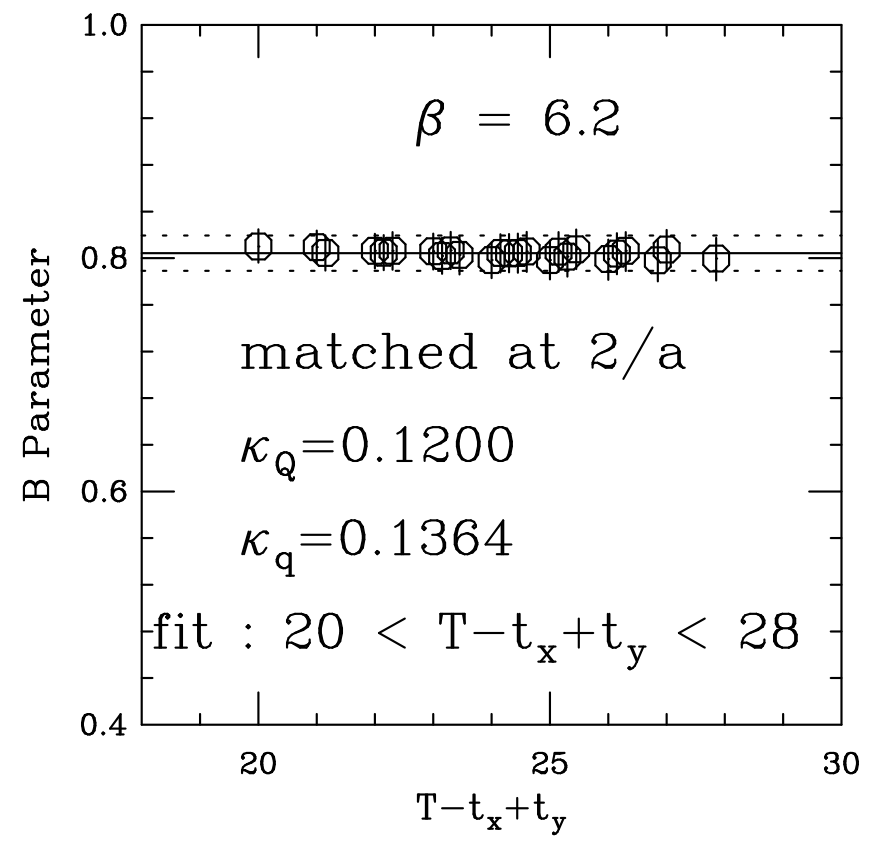

FIG. 1. Plateau for the $B$-parameter of the operator $\mathcal{O}_{q}^{\Delta F=2}$ renormalized at $\mu=2 / a$ in the NDR- $\overline{\mathrm{MS}}$ scheme, for $\beta=6.2$ (i.e. Eq. (26) times $3 /\left(8 Z_{A}^{2}\right)$ ). The correlation function is obtained for $10 \leq t_{y} \leq 14$ and $10 \leq T-t_{x} \leq 14$. Points with the same $T-t_{x}+t_{y}$ are shifted for clarity.
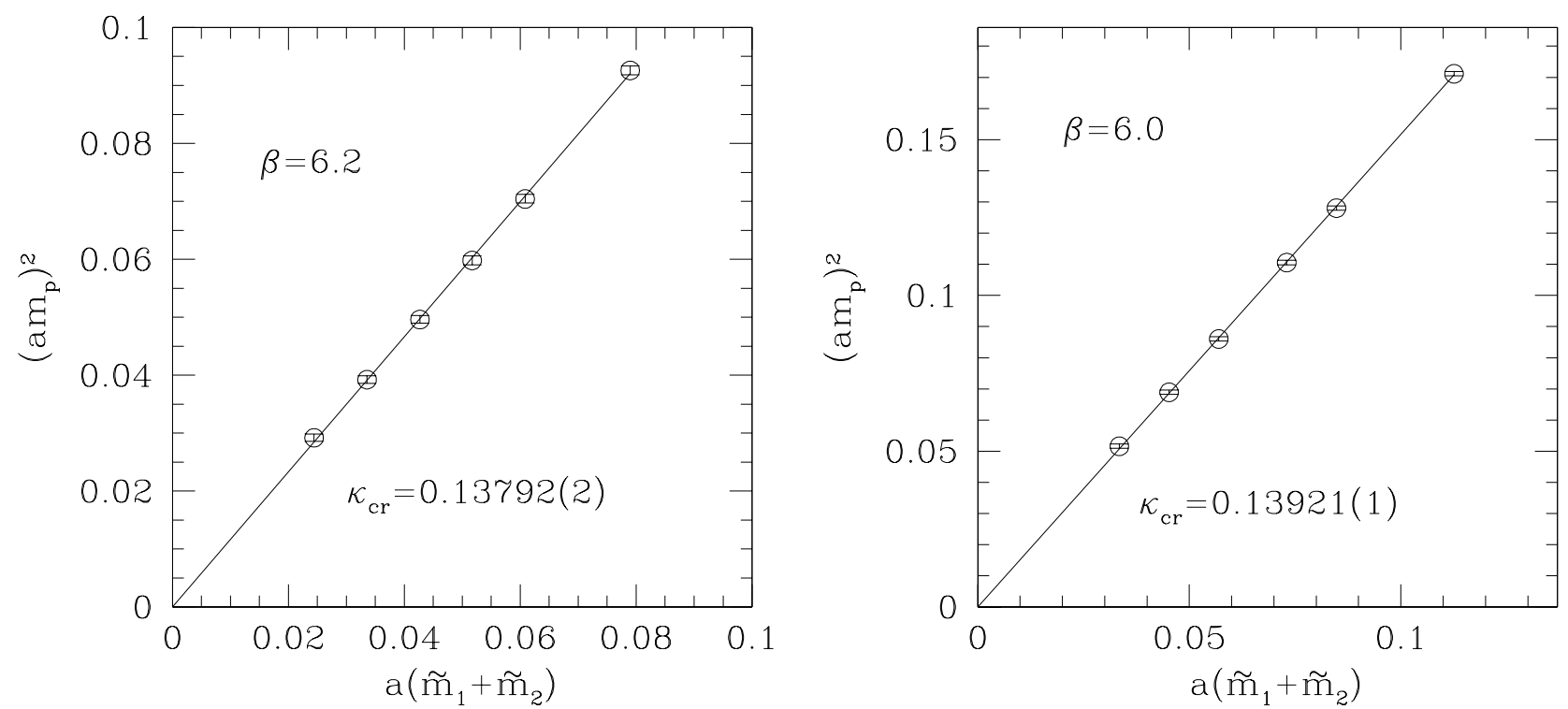

FIG. 2. Fits of the squared, light-light pseudoscalar meson masses versus light-quark mass to the PCAC relation of Eq. (29), at $\beta=6.2$ and 6.0. 

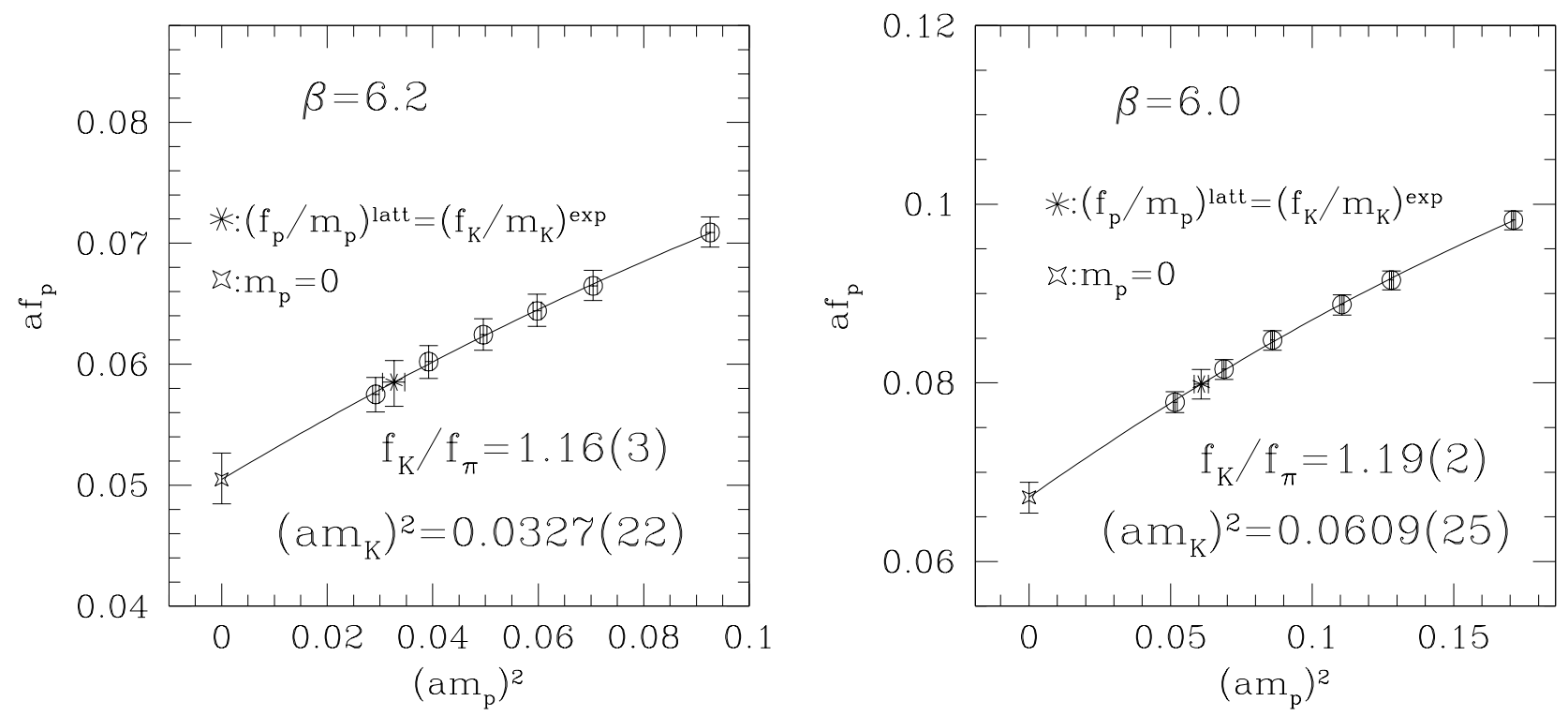

FIG. 3. Interpolation of $a f_{p}$, according to Eq. (31), which is used to determine the scale $a^{-1}$, at $\beta=6.2$ and 6.0 , from $f_{K}$ and $m_{K}$, as described in the text.
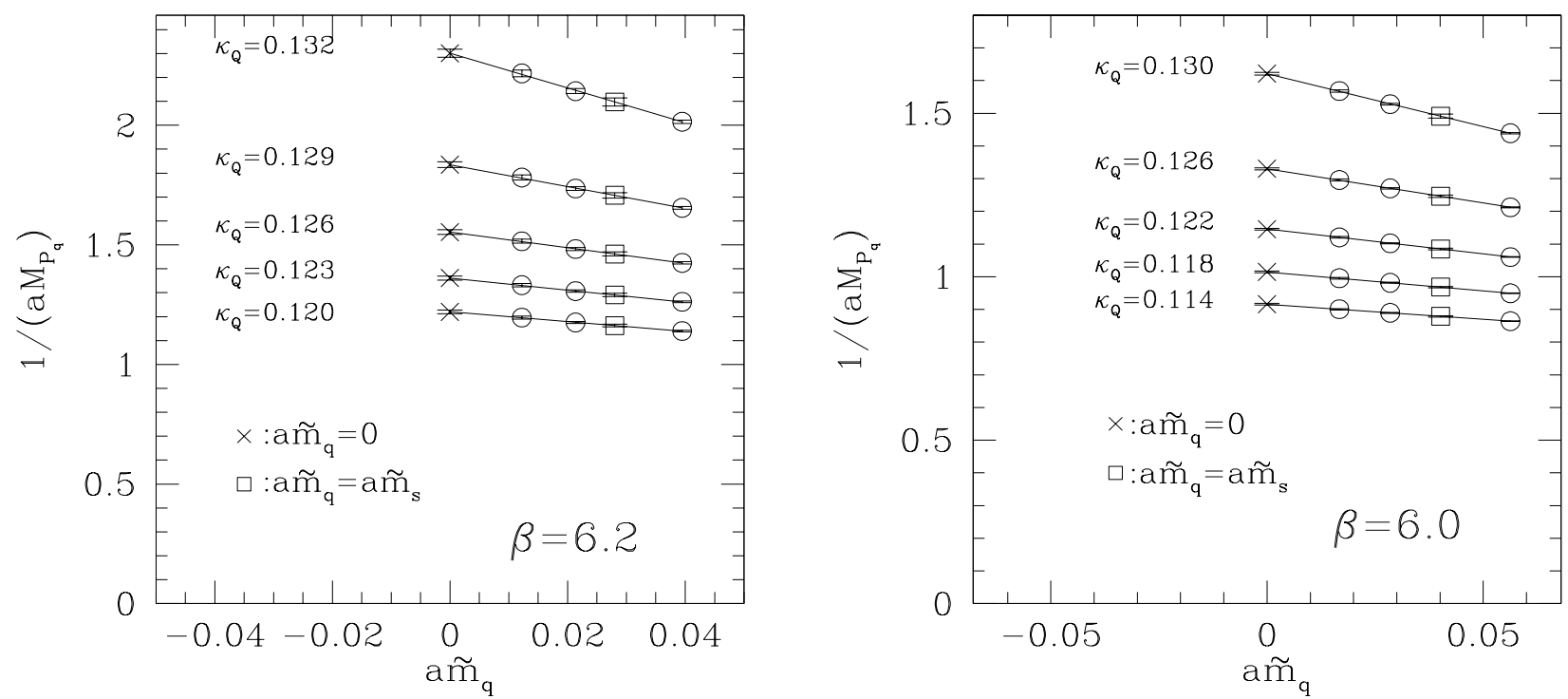

FIG. 4. Light-quark-mass dependence of the heavy-light pseudoscalar meson mass, $M_{P_{q}}$, and extrapolation and interpolation to vanishing quark mass and strange-quark mass at $\beta=6.2$ and 6.0 . 

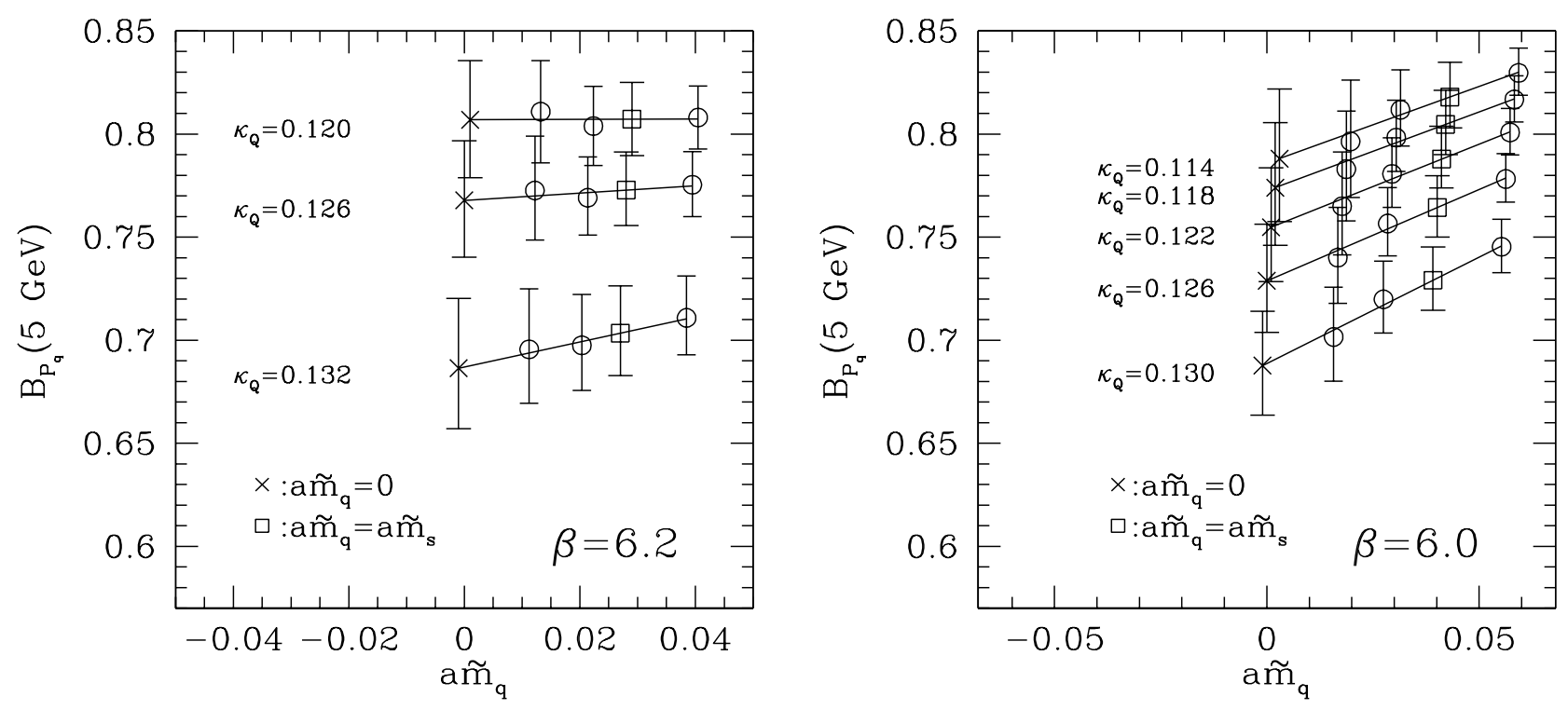

FIG. 5. Light-quark-mass dependence of the heavy-light $B$-parameter, $B_{P_{q}}(5 \mathrm{GeV})$, and extrapolation and interpolation to vanishing quark mass and strange-quark mass at $\beta=6.2$ and 6.0. Points with the same $a \tilde{m}_{q}$ are shifted for clarity.
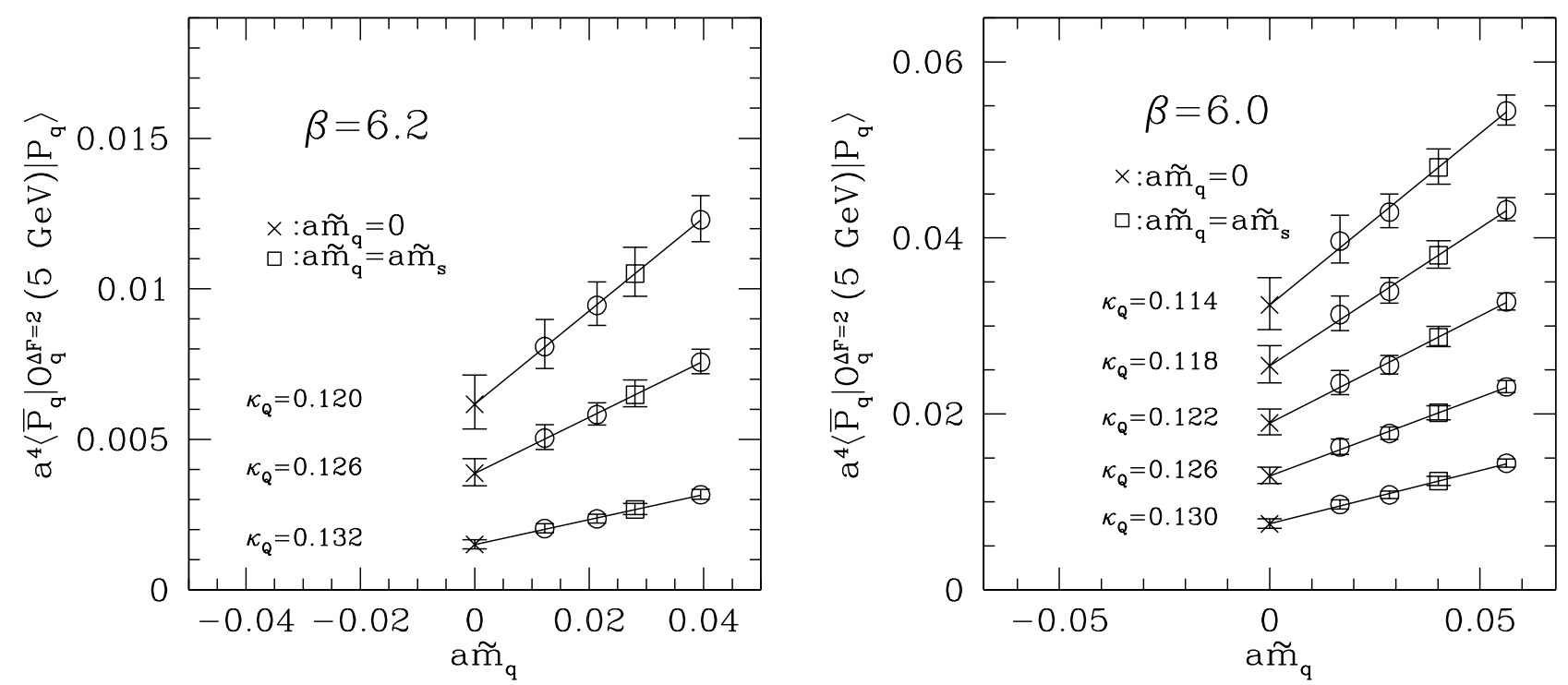

FIG. 6. Light-quark-mass dependence of $\left\langle\bar{P}_{q}\left|\mathcal{O}_{q}^{\Delta F=2}(5 \mathrm{GeV})\right| P_{q}\right\rangle$, and extrapolation and interpolation to vanishing quark mass and strange-quark mass at $\beta=6.2$ and 6.0 . 

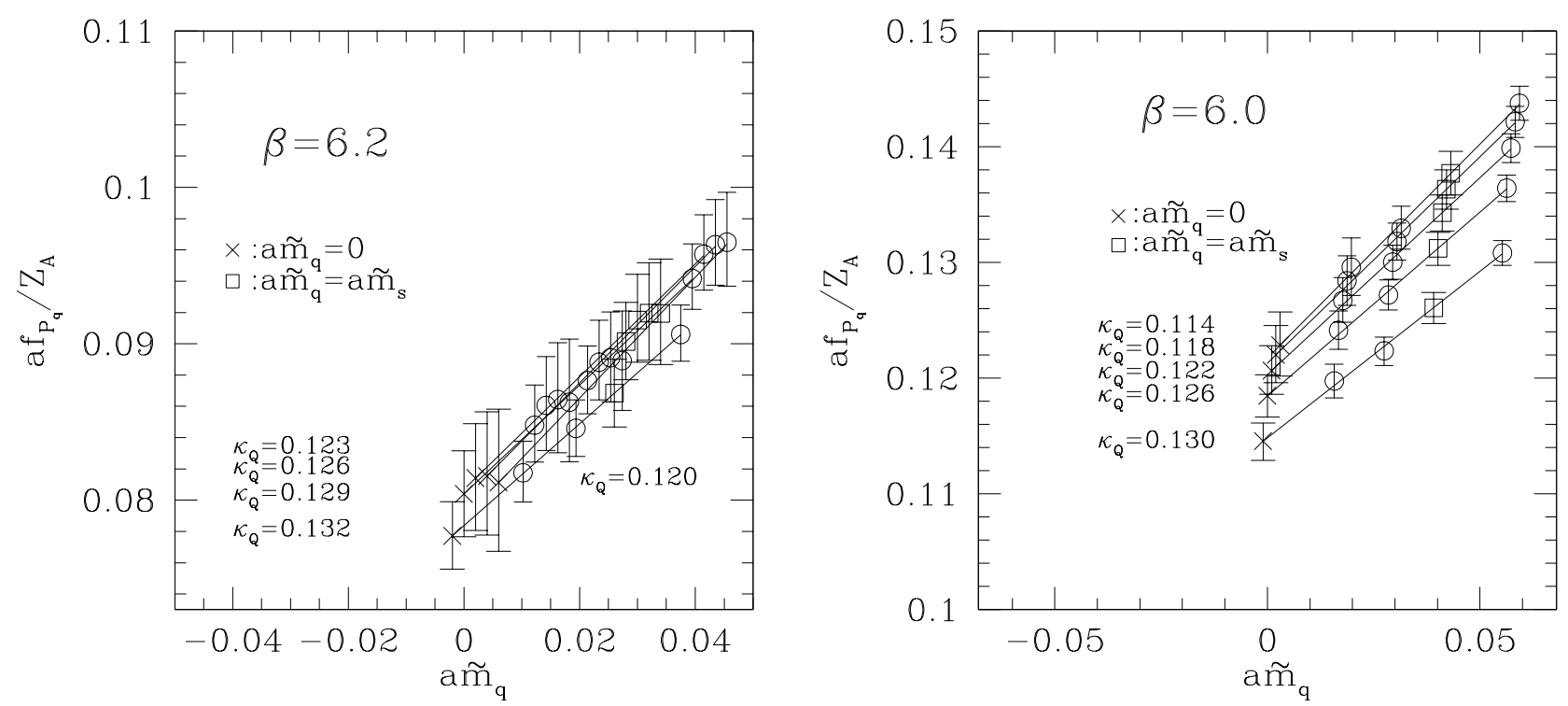

FIG. 7. Light-quark-mass dependence of the heavy-light decay constant, $f_{P_{q}}$, and extrapolation and interpolation to vanishing quark mass and strange-quark mass at $\beta=6.2$ and 6.0. Points with the same $a \tilde{m}_{q}$ are shifted for clarity.

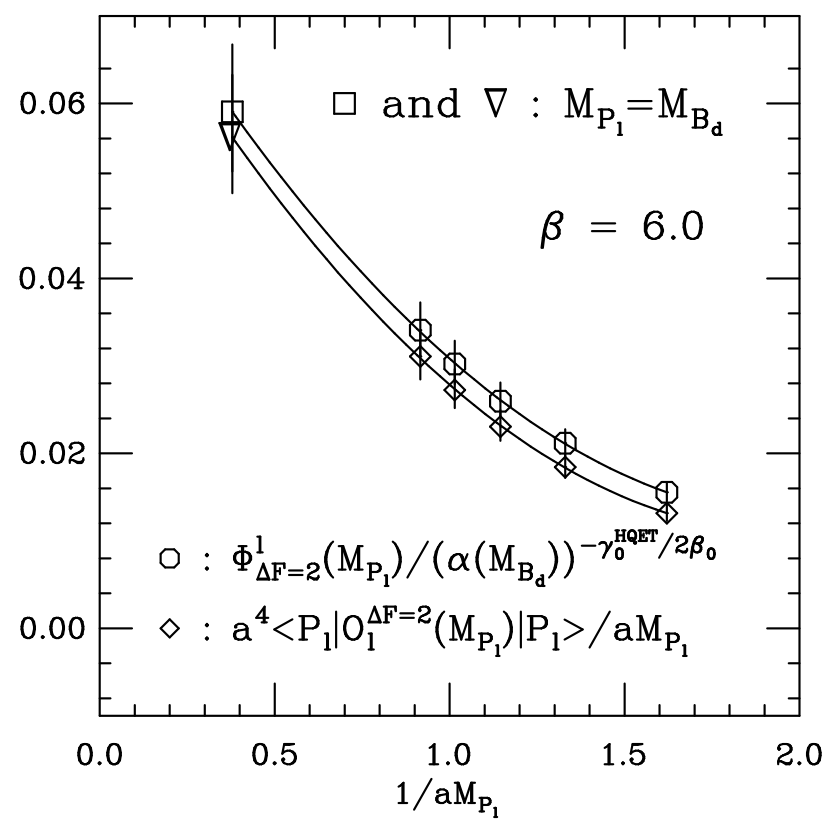

FIG. 8. Influence of leading logarithms on heavy-quark-mass scaling: behavior of $a^{4}\left\langle\bar{P}_{l}\left|\mathcal{O}_{l}^{\Delta F=2}\left(M_{P_{l}}\right)\right| P_{l}\right\rangle / a M_{P_{l}}$ (i.e. logarithms omitted) and of $\Phi_{\Delta F=2}^{l}\left(M_{P_{l}}\right) / \alpha_{s}\left(M_{B_{d}}\right)^{4 / 11}$ (i.e. leading logarithms included) versus $1 /\left(a M_{P_{l}}\right)$. 

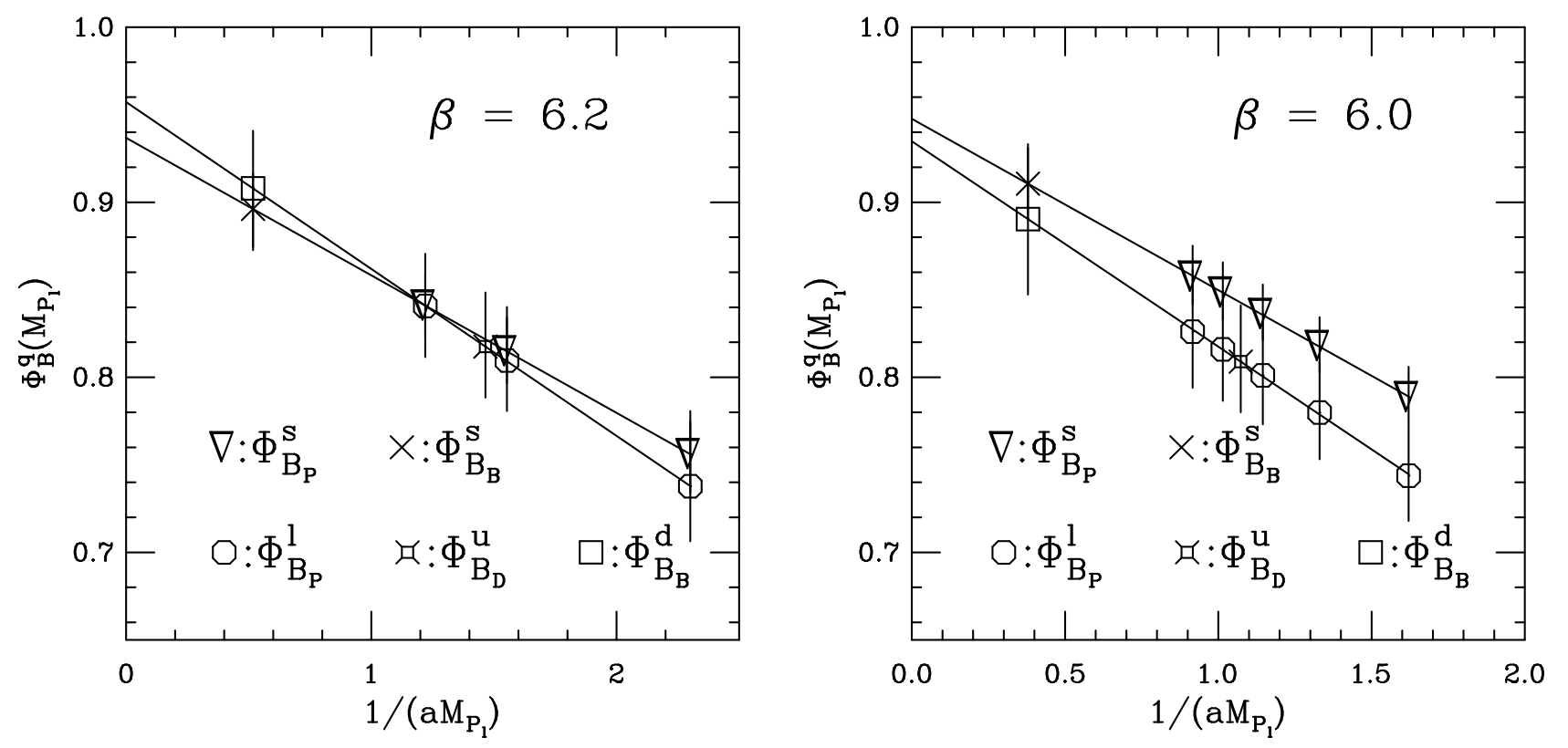

FIG. 9. Lattice results for $X M_{P_{l}}()=\Phi_{B}^{q}\left(M_{P_{l}}\right)$ versus $1 /\left(a M_{P_{l}}\right)$ at $\beta=6.2$ and 6.0 and for $q=s$ and $l$. The solid lines are fits to the linear part of the heavy-quark-mass dependence given in Eq. (36).
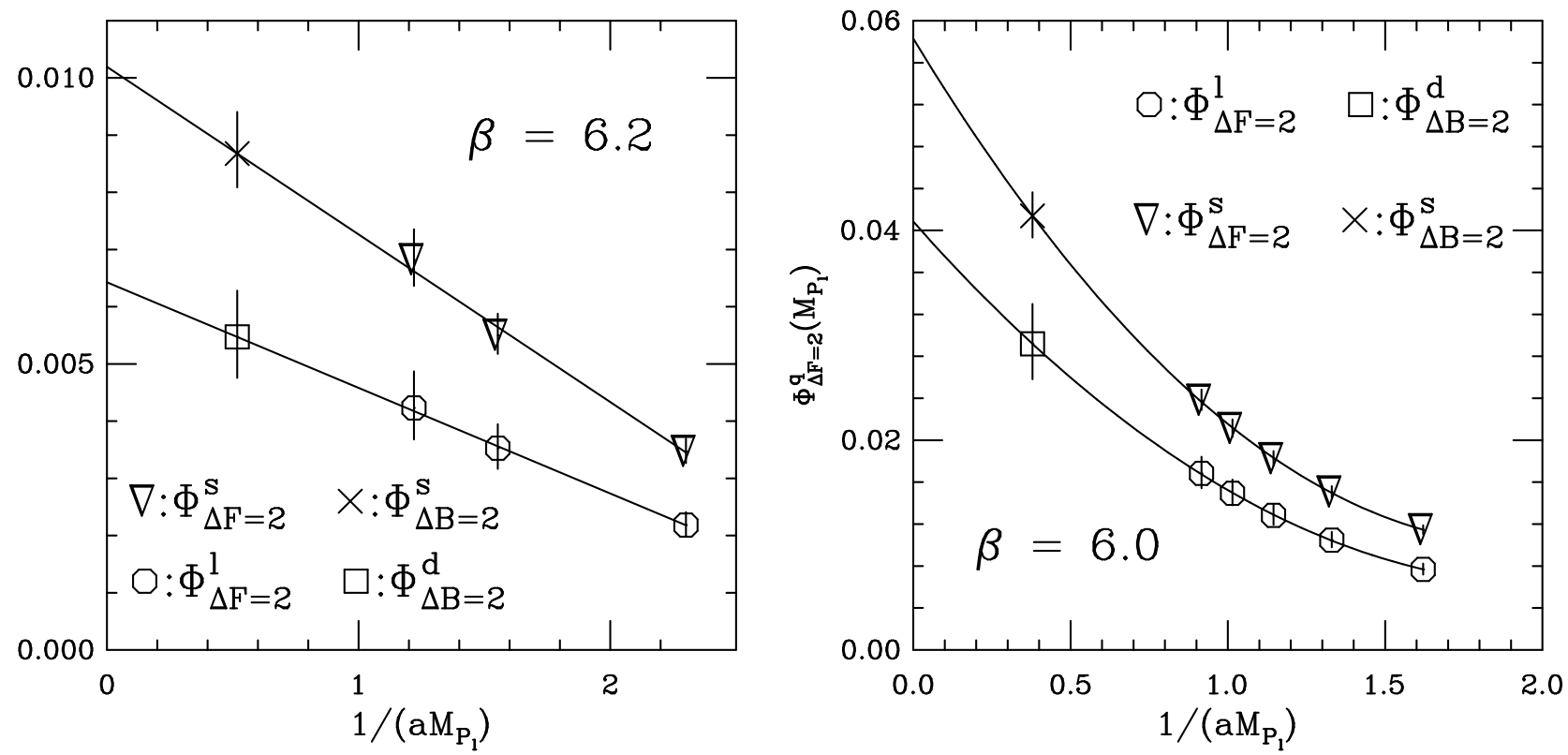

FIG. 10. Lattice results for $X\left(M_{P_{l}}\right)=\Phi_{\Delta F=2}^{q}\left(M_{P_{l}}\right)$ versus $1 /\left(a M_{P_{l}}\right)$ at $\beta=6.2$ and 6.0 and for $q=s$ and $l$. The solid curves are linear and quadratic fits of the heavy-quark-mass dependence given in Eq. (36) at $\beta=6.2$ and $\beta=6.0$, respectively. 

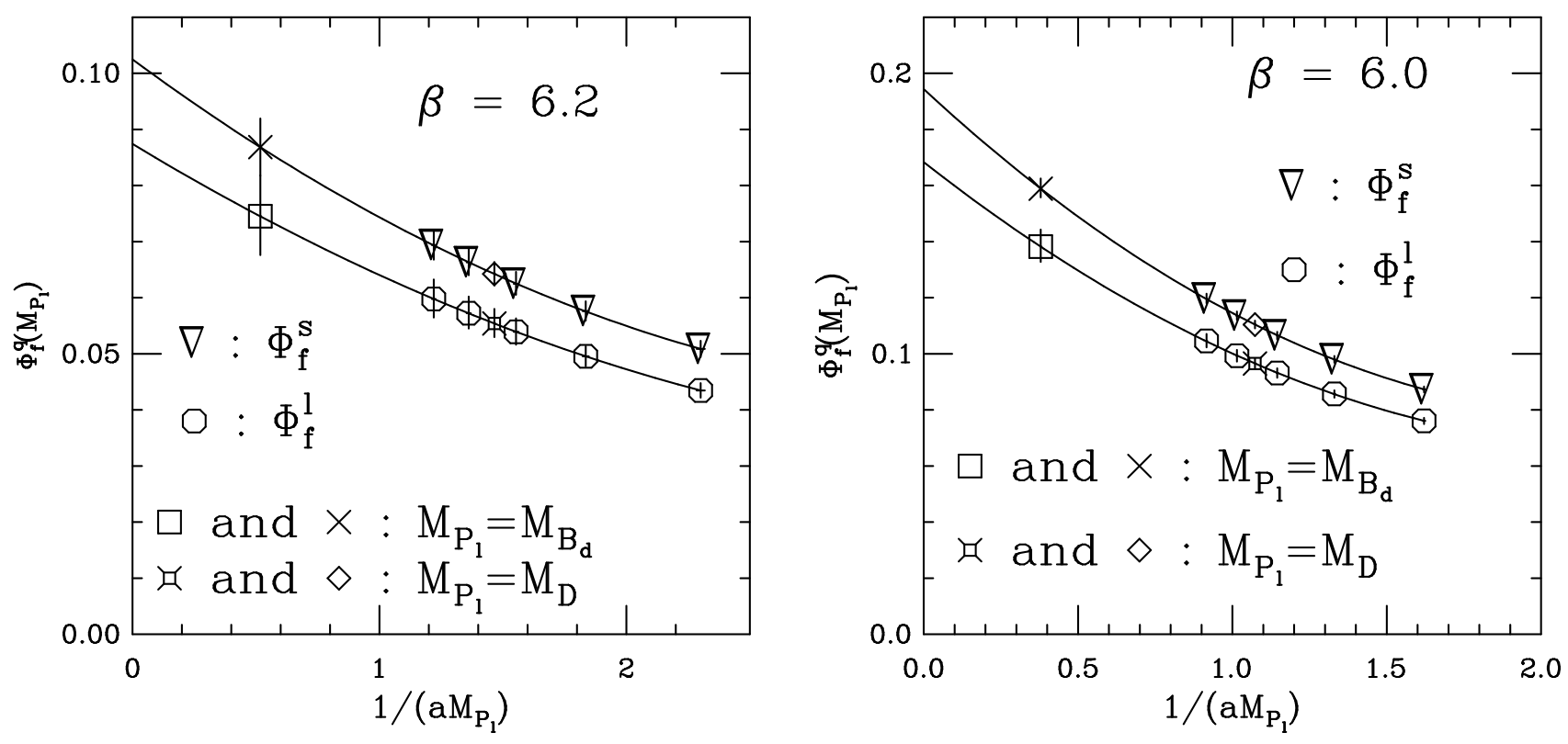

FIG. 11. Lattice results for $X\left(M_{P_{l}}\right)=\Phi_{f}^{q}\left(M_{P_{l}}\right)$ versus $1 /\left(a M_{P_{l}}\right)$ at $\beta=6.2$ and 6.0 and for $q=s$ and $l$. The solid curves are quadratic fits of the heavy-quark-mass dependence given in Eq. (36).
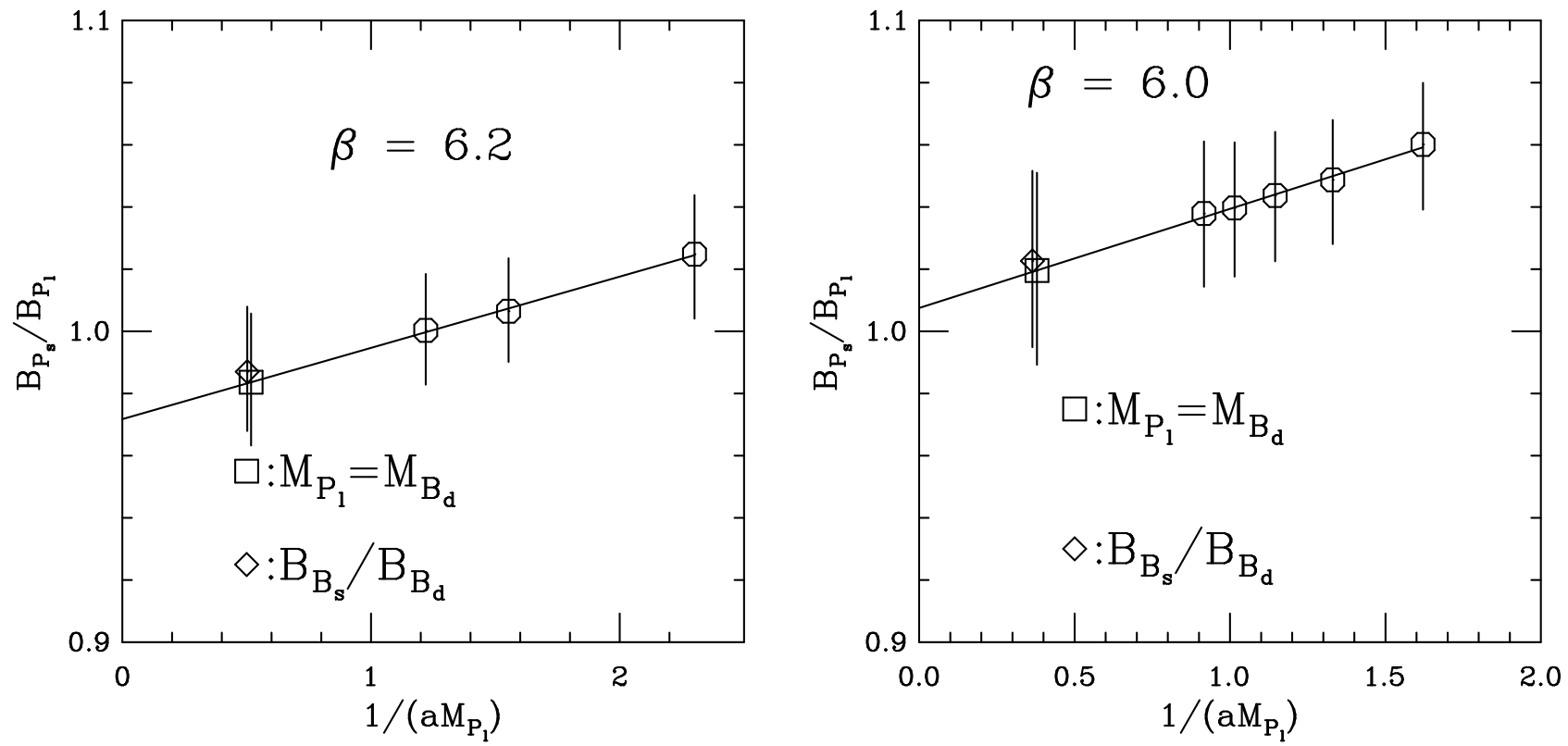

FIG. 12. Lattice results for $X\left(M_{P_{l}}\right)=B_{P_{s}} / B_{P_{l}}$ versus $1 /\left(a M_{P_{l}}\right)$ at $\beta=6.2$ and 6.0 . The solid line is a fit to the linear part of the heavy-quark-mass dependence given in Eq. (36). 

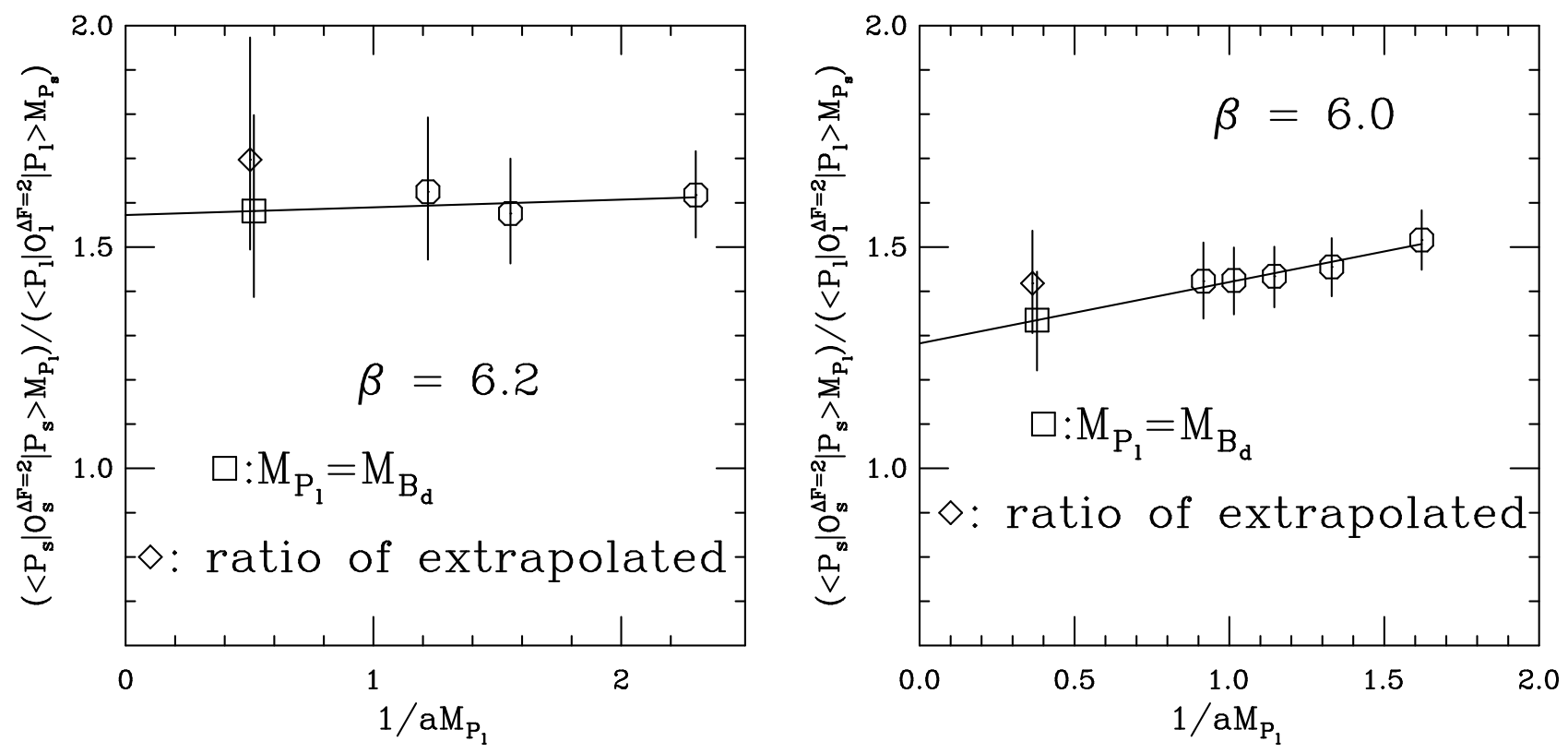

FIG. 13. Lattice results for $X\left(M_{P_{l}}\right)=\left(\left\langle\bar{P}_{s}\left|\mathcal{O}_{s}^{\Delta F=2}\right| P_{s}\right\rangle /\left\langle\bar{P}_{l}\left|\mathcal{O}_{l}^{\Delta F=2}\right| P_{l}\right\rangle\right) \times\left(M_{P_{l}} / M_{P_{s}}\right)$ versus $1 /\left(a M_{P_{l}}\right)$ at $\beta=6.2$ and 6.0 . The solid line is a fit to the linear part of the heavy-quark-mass dependence given in Eq. (36).
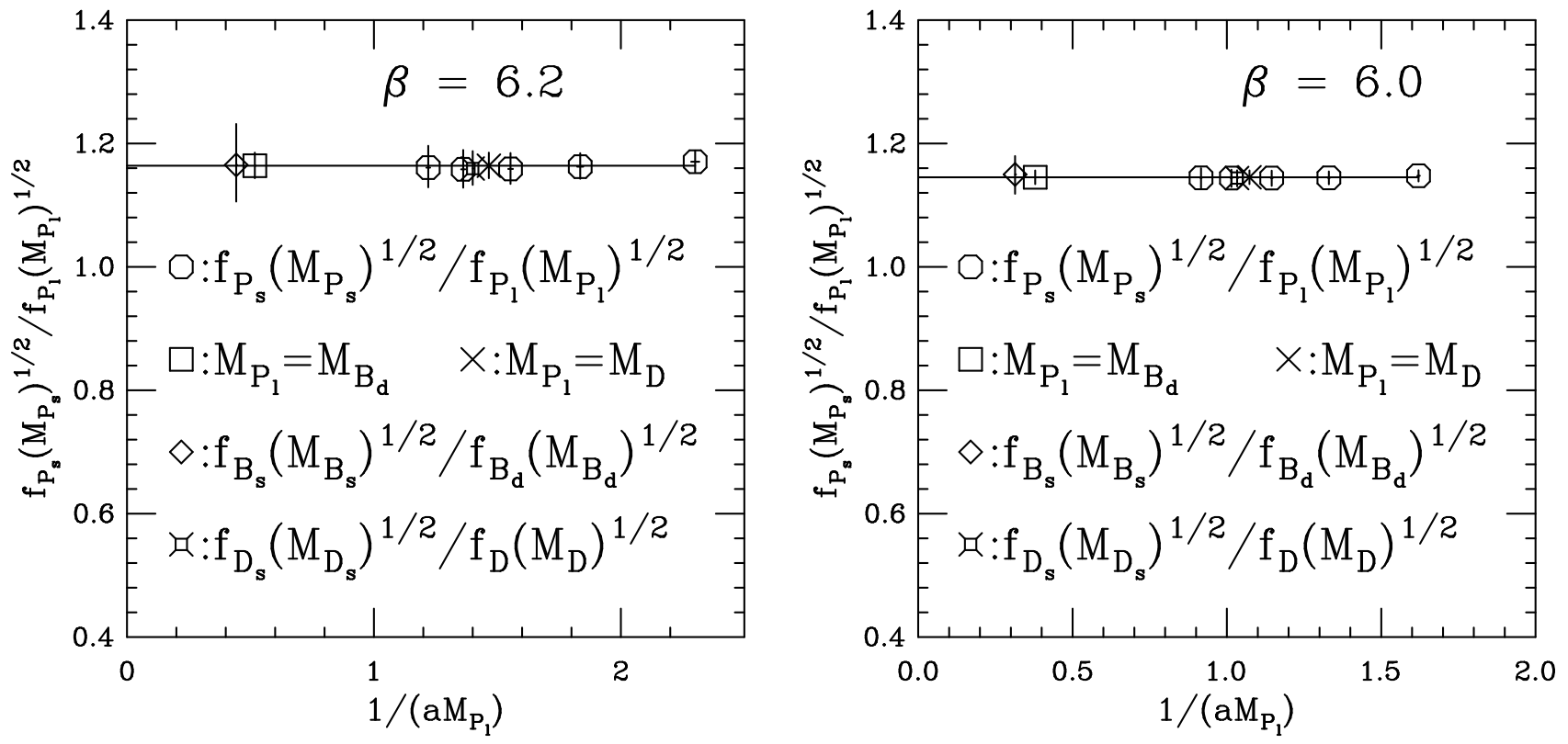

FIG. 14. Lattice results for $X\left(M_{P_{l}}\right)=f_{P_{s}} / f_{P_{l}} \times \sqrt{M_{P_{s}} / M_{P_{l}}}$ versus $1 /\left(a M_{P_{l}}\right)$ at $\beta=6.2$ and 6.0 . The solid line is a fit to the constant part of the heavy-quark-mass dependence given in Eq. (36). 